\title{
Constrained portfolio-consumption strategies with uncertain parameters and borrowing costs
}

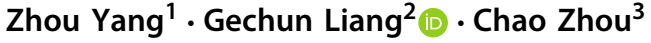

Published online: 26 December 2018

(c) The Author(s) 2018

\begin{abstract}
This paper studies the properties of the optimal portfolio-consumption strategies in a finite horizon robust utility maximization framework with different borrowing and lending rates. In particular, we allow for constraints on both investment and consumption strategies, and model uncertainty on both drift and volatility. With the help of explicit solutions, we quantify the impacts of uncertain market parameters, portfolio-consumption constraints and borrowing costs on the optimal strategies and their time monotone properties.
\end{abstract}

Keywords Robust utility maximization · Explicit solutions · Portfolio-consumption constraints $\cdot$ Different borrowing and lending rates $\cdot$ Model uncertainty

AMS subject classifications $35 \mathrm{R} 60 \cdot 47 \mathrm{~J} 20 \cdot 93 \mathrm{E} 20$

JEL Classifications C61 · G11

\section{Introduction}

One of the fundamental problems in mathematical finance is the construction of investment and consumption strategies $(\pi, c)$ that maximize the expected utility of a risk-averse investor:

\footnotetext{
The authors thank the Editor-in-Chief, the Associate Editor, and the two referees for their valuable comments and suggestions. Yang's work is supported by NNSF of China (Grant No. 11771158, 11801091). Zhou's work is supported by Singapore MOE (Ministry of Education's) AcRF Grant R-146-000-219-112 and R-146-000-255-114.
}

$凶$ Gechun Liang

g.liang@warwick.ac.uk

Zhou Yang

yangzhou@scnu.edu.cn

Chao Zhou

matzc@nus.edu.sg

1 School of Mathematical Sciences, South China Normal University, Guangzhou 510631, China

2 Department of Statistics, University of Warwick, Coventry CV4 7AL, UK

3 Department of Mathematics, Institute of Operations Research and Analytics, and Suzhou Research Institute, National University of Singapore, Singapore, Singapore 


$$
\max _{(\pi, c)} E\left[\int_{0}^{T} U^{c}\left(c_{S}\right) d s+U\left(X_{T}^{\pi, c ; \mu, \sigma}\right)\right],
$$

where $U^{c}(\cdot)$ and $U(\cdot)$ are the utilities of intertemporal consumption $c$ and terminal wealth $X_{T}^{\pi, c ; \mu, \sigma}$, respectively. The market is described by a set of parameters $(\mu, \sigma)$-the drift and volatility of the risky assets, and the investor's utilities are often assumed to admit some homothetic properties (for example, power, logarithm and exponential types). Due to the market incompleteness arising from the randomness of the market parameters and the portfolio constraints, the resulting optimal portfolio is described as the sum of a myopic strategy of Merton's type and a hedging strategy. The latter is used to partially hedge the market risk stemming from the market incompleteness. Both the hedging strategy and the optimal consumption can be described via the solution of a backward stochastic differential equation (see $[7,15])$. However, the solution is in general not explicit, and consequently, there is limited information about the properties of the optimal strategies.

The purpose of this article is to study the properties of the optimal investment and consumption strategies when the investor optimally allocates her wealth among risky and riskless assets and her consumption. Our model takes consideration of several features including model uncertainty, constraints on both investment and consumption strategies, and borrowing costs. Under both power and logarithm utility functions, we characterize the optimal portfolio-consumption strategies and the worst-case market parameters using the solutions of nonlinear ODEs, and furthermore, derive their explicit solutions in one-dimensional setting. The explicit forms further allow us to study the impacts of uncertain market parameters, portfolio-consumption constraints and different borrowing and lending rates on the optimal strategies and their time monotone properties.

In the vast majority of the literature, it is often assumed that the investor has a perfect knowledge of the market parameters, and is able to select her portfolio-consumption strategies without any constraints. However, constraints such as prohibition of short selling risky assets and the subsistence consumption are ubiquitous in reality. On the other hand, the paradigm of expected utility clearly has some deficiencies: it is not satisfactory in dealing with model uncertainty as predicted by the famous Ellsberg paradox. For the above reasons, it is desirable to take constraints on the portfolio-consumption strategies and uncertainty about the market parameters into account when studying the optimal strategies. We argue that the portfolioconsumption strategies must stay in a closed and convex set, and there are lots of probability models to describe the market, but none of them are really precise enough. This leads us to consider the so called robust utility maximization for which the investor worries about the worst-case scenario, ${ }^{1}$ and as opposed to (1), we solve the following maxmin problem

$$
\max _{(\pi, c) \in \mathcal{B}} \min _{(\mu, \sigma) \in \mathcal{A}} E\left[\int_{0}^{T} U^{c}\left(c_{s}\right) d s+U\left(X_{T}^{\pi, c ; \mu, \sigma}\right)\right],
$$

for an investor with power or logarithm type utilities on both intertemporal consumption and terminal wealth. See (4) and (5) for further details.

As a first contribution, we show that the functions used to construct the value processes for power and logarithm utilities [see $F_{P}$ and $F_{L}$ in (8)] admit saddle points (Lemma 3.1). The saddle points in turn characterize locally the optimal portfolio-consumption strategies and the worst-case parameters. Since the constraint set for the portfolios-consumption strategies may not be compact, it is not even clear ex ante whether a saddle point exists or not. We tackle

\footnotetext{
1 Note that the worst-case scenario approach implies that the investor behaves too conservative, which is not always the case in reality. Recently, an interesting paper [14] casts the investor having moderate risks and uncertainty aversions. We refer to [14] for a further discussion of this approach.
} 
the problem by an approximation procedure using a sequence of saddle points in compact sets to construct a saddle point in the non-compact constraint set. We further characterize the optimal strategies using the solutions of nonlinear ODEs in Theorem 3.2 (for power utility) and Theorem 3.3 (for logarithm utility). We show that even with random market parameters and portfolio-consumption constraints, the optimal strategies are however deterministic in a robust utility framework. It is due to the fact that when the investor worries about the worstcase scenario, the optimal strategies are given via a deterministic saddle point and the solution of an associated nonlinear ODE. Eventually, this leads the investor to implement myopic strategies of Merton's type to optimize her portfolios as in a complete market. Thus, there is no need for her to enforce the hedging strategy as opposed to the incomplete market situation. A similar phenomenon also occurs in [23], where the authors considered a market driven by Lévy processes with uncertain parameters but without consumption and borrowing costs.

Furthermore, in one-dimensional setting we obtain the optimal portfolio-consumption strategies and the worst-case parameters both in closed forms. Closed-form solutions seldom exist except for the standard Merton's model with constant market parameters without portfolio-consumption constraints. We find that the explicit solutions still exist for both power and logarithm utility functions in the general framework incorporating model uncertainty, constraints on both investment and consumption strategies, and borrowing costs.

As the first example, when the uncertain market parameters stay in an interval set, we obtain a classification of the optimal portfolio strategies in terms of borrowing and lending rates as well as the uncertain market parameters. We show that (1) when the investor is optimistic about the market, meaning that her worst estimation of the stock's return is still better than the borrowing rate, she will implement a borrow-to-buy strategy to borrow as much as possible to approach the optimal strategy without constraint. (2) When her worst estimation of the stock's return is between the borrowing and lending rates, neither borrowing nor lending are attractive, and the investor will simply put all her money in the stock, i.e. performing a full-position strategy. (3) When the lending rate is between the best and worst estimations of the stock's return, the investor will simply put all her money in the bank account, i.e. performing a no-trading strategy. (4) When the investor is pessimistic about the market, meaning that her best estimation of the stock's return is still lower than the lending rate, she will implement a shortsale strategy to short sell the stock as much as possible. See Theorem 4.2 for further details.

As the second example, when the uncertain drift and volatility are correlated, we further show that the saddle point may become an interior point of the uncertain parameter set. The worst-case parameters are then given through the explicit interior saddle point, as opposed to the bang-bang type of saddle points in the existing literature. As a result, the optimal portfolio strategy is also given through the interior saddle point, albeit still in Merton's type. See Theorem 4.4 for further details.

The explicit solutions further allows us, for the first time, to give a systematic study of the consumption plans in various situations. We argue that the consumption should stay above a minimum level for subsistence purpose, and be dominated by a reasonable upper bound for the sake of future consumption and investment. We show that the investor's optimal consumption will degenerate to a deterministic process when she worries about the worstcase market scenario (see Theorem 4.5 for the power case and Theorem 4.7 for the logarithm case). By virtue of the closed form solutions, we are able to obtain the time monotone properties of the optimal consumption plan (see Proposition 4.6 and Theorem 4.7), and quantify the impacts of different parameters (e.g. borrowing rate, uncertain market parameters and portfolio-consumption constraints) on the optimal consumption plan (see Propositions 5.1 and 5.2). 
One of the striking results is that, for the power utility case, the optimal consumption is not necessarily increasing or decreasing when the investor lifts her upper bound for consumption. This is because the investor needs to balance her current consumption and future consumption and investment when she optimizes her consumption plans. Increasing the upper bound of consumption means the investor would consume in a larger constraint set in the future, and increase the weight of her future utility, thus the investor might decrease her current consumption level. On the other hand, lifting the upper bound for consumption also means a larger constraint set from which the investor makes her current consumption decisions, and in turn her current consumption level might increase. This two contradicting factors will offset their impacts by each other, and result in a non-monotone relationship of optimal consumption with respect to the upper bound of consumption plans.

Turning to the literature, optimal portfolio-consumption problems in continuous time were first studied by Merton in 1970s (see [22] for a summary). In a sequence of papers [16,17] and [19], the authors developed and generalized Merton's model. In particular, [19] is one of the first arguing that the consumption must always be above a certain subsistence level, and sometimes neither borrowing nor shortsale are allowed for trading stocks, so they imposed constraints on both consumption and investment. Following this work, the optimal consumption with constraints was further studied in [6,27], and more recently in [18,31] in a complete market setting with constant market parameters. On the other hand, $[8,30,32]$ and [33] among others studied constrained investment problems for models of varying generality.

Equal borrowing and lending rates is often assumed in the literature, and as a consequence, the wealth equation is always linear. However, it is argued in [1] that such an assumption stands in contrast with reality. Subsequently, [11] introduced the borrowing cost for the utility maximization problem, and more recently in [3], the authors took borrowing costs into account in an optimal credit investment problem.

The early development of model uncertainty went back to [28] where the authors considered a worst-case risk management problem. Robust utility maximization in mathematical finance started with [4,13] and [26], which mainly dealt with drift uncertainty. The problem of volatility uncertainty is much harder, and has been treated via various mathematical tools. To name a few, duality method was used in [9] where the uncertainty is specified by a family of semimartingales laws. $G$-expectation was employed in [12] in a stochastic volatility model to treat uncertain correlations. In contrast, [21] studied the robust utility maximization problem under volatility uncertainty via second-order backward stochastic differential equations, and [29] considered uncertain drift and volatility using mixed strategies and derived an explicit solution in a non-traded asset setting. More recently, the results have been further generalized in [23] to include drift, volatility and jump uncertainty, which are parameterized by a set of Lévy triples. However, consumption is not considered in the above works. Two exceptions are [20] and more recently [2], where the authors worked in a similar framework to our model, but portfolio-consumption constraints are not treated in those papers.

In summary, it seems the existing literature mainly focuses on the investment-consumption models with only parts of the above features: either with portfolio constraints and market uncertainty or with consumption constraints and borrowing costs. Although many elegant mathematical results are achieved in these papers, explicit solutions and the properties of the optimal strategies rarely exist except for some special cases. In particular, consumption constraints make it difficult to obtain explicit solutions, and almost all of the explicit solutions with consumption constraints are in the framework of infinite horizon (see [6], [19] and [27]).

In contrast, our paper systematically studies constrained portfolio-consumption strategies under model uncertainty and borrowing costs in a finite horizon, and quantifies their impacts on the optimal strategies. We obtain explicit solutions and properties of the optimal strategies. 
Although explicit solutions are derived under one risky asset setting, our method can be applied to study the multiple risky assets setting as in [23], and similar results will still hold, albeit with more complicated situations.

The paper is organized as follows. Section 2 presents a robust utility maximization model subject to borrowing costs and portfolio-consumption constraints in a multiple risky assets setting. Section 3 solves the associated maxmin problem via a martingale argument, and characterizes the optimal portfolio-consumption strategies and the worst-case market parameters via the solutions of nonlinear ODEs. Section 4 further obtains their closed form solutions in a single risky asset setting with different uncertain parameter sets. Section 5 studies the impacts of the various model parameters on the optimal strategies and the worst-case parameters. The proof of explicit solutions is given in the "Appendix".

\section{The utility maximization model}

\subsection{Uncertain parameters and borrowing costs}

Let $d$ and $d^{\prime}$ be two positive integers. Let $W$ be a standard $d^{\prime}$-dimensional Brownian motion defined on a complete probability space $(\Omega, \mathcal{F}, \mathbb{P})$, and $\mathbb{F}:=\left\{\mathcal{F}_{t}\right\}_{t \geq 0}$ be the augmented filtration generated by $W$. The market consists of $d$ risky assets and a riskless bank account. The price processes of the risky assets $S_{i}, 1 \leq i \leq d$, solve

$$
d S_{i, s}=\mu_{i, s} S_{i, s} d s+\Sigma_{j=1}^{d^{\prime}} \sigma_{s}^{i j} S_{i, s} d W_{j, s}
$$

for $s \geq 0$, where $\mu:=\left(\mu_{1}, \ldots, \mu_{d}\right)^{\mathrm{T}}$ and $\Sigma:=\left(\sigma^{i j}\right)_{d \times d^{\prime}}$ represent the drift and volatility of the risky assets, respectively.

Consider a small investor in this market. She trades both the risky assets and riskless bank account, yet she has limited information about the risky assets' parameters $(\mu, \Sigma)$. The uncertainty about drift and volatility of the risky assets is parameterized by a nonempty set with the form

$$
\begin{aligned}
\mathcal{B}= & \left\{\left(\mu_{s}, \Sigma_{s}\right)_{s \geq 0}:(\mu, \Sigma) \text { are } \mathbb{F}\right. \text {-progressively measurable, and } \\
& \left.\left(\mu_{s}, \Sigma_{s} \Sigma_{s}^{\mathrm{T}}\right) \in \mathbb{B}, \mathbb{P} \otimes d s \text {-a.e. }\right\},
\end{aligned}
$$

where $\mathbb{B}$ is a convex and compact subset of $\mathbb{R}^{d} \times \mathcal{S}_{+}^{d}$, with $\mathcal{S}_{+}^{d}$ being the set of $d \times d$ positive semi-definite real symmetric matrixes. We also assume that $\mathbb{B}$ contains at least one element $(\mu, \Sigma)$ such that $\Sigma \Sigma^{\mathrm{T}}$ is positive definite. The area of the set $\mathbb{B}$ indicates the amount of uncertainty. The larger the area, the larger becomes the set of alternative models. The investor will then become more uncertain about the model parameters.

In terms of the bank account $B$, the standard assumption of equal borrowing and lending rates is in contrast with empirical evidence (see [1]). In reality, there always exists a spread between borrowing and lending rates. Let $R$ and $r$ be the constant borrowing and lending rates, respectively. When $B$ is positive, the investor lends with rate $r$. When $B$ is negative, the investor borrows with rate $R$. It is nature to assume that $R \geq r$. Consequently, the bank account $B$ follows

$$
d B_{s}=\left(r B_{s}^{+}-R B_{s}^{-}\right) d s,
$$

where $x^{+}=\max \{0, x\}, x^{-}=\max \{0,-x\}$. Note that $r B_{s}^{+}-R B_{s}^{-}=r B_{s}-(R-r) B_{s}^{-}$, and therefore the spread $(R-r)$ represents the borrowing cost of the investor. The larger the 
spread, the more borrowing cost the investor has to bear. In the next section, we shall see the introduction of borrowing cost leads to a nonlinear wealth equation, which is concave in the portfolio strategies.

\subsection{Portfolio and consumption constraints}

Let $T>0$ represent the trading horizon, and suppose that the investor has an initial wealth $x>0$. Let $\pi$ be the proportion of her wealth invested in the risky assets, $c$ be her consumption rate proportional to her wealth, and $X^{x ; \pi, c, \mu, \Sigma}$ be the wealth process with initial value $x$, portfolio-consumption strategies $(\pi, c)$ and parameters $(\mu, \Sigma)$. Using (2) and (3), it follows from the self-financing condition that

$$
\begin{aligned}
X_{s}^{x ; \pi, c, \mu, \Sigma}= & x+\int_{0}^{s}\left[\mu_{u}^{\mathrm{T}} \pi_{u}+r\left(1-1_{d}^{\mathrm{T}} \pi_{u}\right)-(R-r)\left(1-1_{d}^{\mathrm{T}} \pi_{u}\right)^{-}-c_{u}\right] X_{u}^{x ; \pi, c, \mu, \Sigma} d u \\
& +\int_{0}^{s} X_{u}^{x ; \pi, c, \mu, \Sigma} \pi_{u}^{\mathrm{T}} \Sigma_{u} d W_{u}, \quad s \in[0, T] .
\end{aligned}
$$

Note that with the borrowing cost, the drift of the wealth equation is no longer linear but concave in the portfolio strategy $\pi$ in the case of $R>r$.

The investor will select her portfolio-consumption strategies from the the following admissible set with constraints on both portfolio and consumption:

$$
\begin{aligned}
\mathcal{A}=\{ & \left(\pi_{s}, c_{s}\right)_{s \geq 0}:(\pi, c) \text { are } \mathbb{F} \text {-progressively measurable, }\left(\pi_{s}, c_{s}\right) \in \mathbb{A}, \mathbb{P} \otimes d s \text {-a.e., } \\
& \left.\int_{0}^{T}\left(\left|\pi_{s}\right|^{2}+c_{s}\right) d s<+\infty, \text { and } X^{x ; \pi, c, \mu, \Sigma} \text { satisfies the condition }(\mathrm{H})\right\},
\end{aligned}
$$

where $\mathbb{A}$ is a convex and closed subset of $\mathbb{R}^{d+1}$ satisfying that $c \geq 0$. The integrability condition on $(\pi, c)$ is to guarantee that the wealth process is well defined, while the condition (H) imposed on the wealth process $X^{x ; \pi, c, \mu, \Sigma}$ depends on the utility maximization problem that we want to solve, and will be specified in (7) in the next section.

One typical example of the constraint set is $\mathbb{A}=\bigotimes_{i=1}^{d}\left[\underline{\pi}_{i}, \bar{\pi}_{i}\right] \times[\underline{c}, \bar{c}]$, where $\underline{\pi}_{i}, \bar{\pi}_{i}, \underline{c}, \bar{c}$ are constants satisfying $-\infty \leq \underline{\pi}_{i} \leq 0,1 \leq \bar{\pi}_{i} \leq+\infty, 0 \leq \underline{c} \leq \bar{c} \leq+\infty$ for $i=1, \ldots, d$. Then, the portfolio constraint cube $\bigotimes_{i=1}^{d}\left[\underline{\pi}_{i}, \bar{\pi}_{i}\right]$ has the following financial interpretations: $\left(\sum_{i=1}^{d} \bar{\pi}_{i}-1\right)$ represents the maximum proportion of wealth that the investor is allowed to borrow to invest in the risky assets; $\left(-\sum_{i=1}^{d} \underline{\pi}_{i}\right)$ represents the largest shortsale position that the investor is allowed to take; $\pi_{i}=0$ means prohibition of shortsale the $i$ th risky asset; $\bar{\pi}_{i}=1$ means prohibition of borrowing to invest in the $i$ th risky asset; and $-\underline{\pi}_{i}=\bar{\pi}_{i}=+\infty$ means no portfolio constrains on the $i$ th risky asset. Moreover, the consumption constraint $[\underline{c}, \bar{c}]$ means that the investor should keep a minimal consumption level $\underline{c}$ for subsistence purpose, and at the same time, her consumption is also controlled by an upper bound $\bar{c}$ for the sake of future consumption and investment.

\subsection{The robust utility maximization problem}

The investor has utilities of both intertemporal consumption and terminal wealth. Given a portfolio-consumption strategy $(\pi, c) \in \mathcal{A}$, her expected utility is defined as 


$$
\begin{aligned}
& \mathcal{J}_{i}(x ; \pi, c, \mu, \Sigma) \\
& \quad:=\mathbb{E}\left[\int_{0}^{T} \lambda e^{-\rho s} U_{i}^{c}\left(c_{s} X_{s}^{x ; \pi, c, \mu, \Sigma}\right) d s+e^{-\rho T} U_{i}\left(X_{T}^{x ; \pi, c, \mu, \Sigma}\right)\right], \quad i=P, L,
\end{aligned}
$$

where $P, L$ represents, respectively, the power and logarithm utility functions, i.e. $U_{P}^{c}(x)=$ $U_{P}(x)=\frac{1}{p} x^{p}$ with $p \in(-\infty, 0) \cup(0,1)$, and $U_{L}^{c}(x)=U_{L}(x)=\ln x$. Herein, $\lambda \geq 0$ represents the weight of the intertemporal consumption relative to the final bequest at maturity $T$, and $\rho \geq 0$ represents the discount factor.

Since the investor is uncertain about the model parameters $(\mu, \Sigma)$, she will seek for an optimal portfolio-consumption strategy that is least affected by model uncertainty. In anticipation of the worst-case scenario, she solves the following maxmin problem: Find $\left(\pi^{*}, c^{*}\right) \in \mathcal{A}$ and $\left(\mu^{*}, \Sigma^{*}\right) \in \mathcal{B}$ such that

$$
J_{i}(x):=\sup _{(\pi, c) \in \mathcal{A}} \inf _{(\mu, \Sigma) \in \mathcal{B}} \mathcal{J}_{i}(x ; \pi, c, \mu, \Sigma)=\mathcal{J}_{i}\left(x ; \pi^{*}, c^{*}, \mu^{*}, \Sigma^{*}\right), \quad i=P, L,
$$

where $J_{i}(\cdot)$ is the value function of the maxmin problem (5), i.e. the maximum worst-case expected utility.

To robustify the optimal portfolio-consumption strategy, the inner part of the above optimization problem is played by a so called mother nature who acts maliciously to minimize the expected utility by choosing the worst-case scenario, whereas the investor aims to select the best strategy that is least affected by the mother nature's choice. For this reason, the maxmin problem (5) is also dubbed as the robust utility maximization problem in the literature (see [23] for example).

To solve the value function of the robust utility maximization problem (5) and its corresponding worst-case parameters and optimal portfolio-consumption strategies, we look for a saddle point strategy $\left\{\left(\pi^{*}, c^{*}\right),\left(\mu^{*}, \Sigma^{*}\right)\right\}$ of the expected utility $\mathcal{J}_{i}(x ; \pi, c, \mu, \Sigma)$ such that

$$
\mathcal{J}_{i}\left(x ; \pi, c, \mu^{*}, \Sigma^{*}\right) \leq \mathcal{J}_{i}\left(x ; \pi^{*}, c^{*}, \mu^{*}, \Sigma^{*}\right) \leq \mathcal{J}_{i}\left(x ; \pi^{*}, c^{*}, \mu, \Sigma\right)
$$

for any admissible $(\pi, c) \in \mathcal{A}$ and $(\mu, \Sigma) \in \mathcal{B}$. Then, it follows that

$$
\begin{aligned}
\sup _{(\pi, c) \in \mathcal{A}} \inf _{(\mu, \Sigma) \in \mathcal{B}} \mathcal{J}_{i}(x ; \pi, c, \mu, \Sigma) & =\mathcal{J}_{i}\left(x ; \pi^{*}, c^{*}, \mu^{*}, \Sigma^{*}\right) \\
& =\inf _{(\mu, \Sigma) \in \mathcal{B}} \sup _{(\pi, c) \in \mathcal{A}} \mathcal{J}_{i}(x ; \pi, c, \mu, \Sigma),
\end{aligned}
$$

and consequently, $J_{i}(x)=\mathcal{J}_{i}\left(x ; \pi^{*}, c^{*}, \mu^{*}, \Sigma^{*}\right)$ is the value function of the maxmin problem (5), with $\left(\mu^{*}, \Sigma^{*}\right)$ and $\left(\pi^{*}, c^{*}\right)$ as the worst-case parameters and the optimal portfolio-consumption strategies, respectively.

To close this section, we further specify the condition (H) in the admissible set $\mathcal{A}$ associated with the maxmin problem (5):

$$
\begin{aligned}
\text { Condition }(\mathrm{H}):= & \left\{E\left[\int_{0}^{T} U_{i}^{c}\left(c_{s} X_{s}^{x ; \pi, c, \mu, \Sigma}\right) d s\right]<+\infty ; \text { and the family } U_{i}\left(X_{\tau}^{x ; \pi, c, \mu, \Sigma}\right),\right. \\
& \text { for } \tau \in[0, T] \text { as an } \mathbb{F} \text {-stopping time, is uniformly integrable }\}
\end{aligned}
$$

The integrability condition imposed on $U_{i}\left(X^{x ; \pi, c, \mu, \Sigma}\right)$ is to include unbounded portfolio and consumption strategies. This condition is also called Class (D) condition and appears in [7], where the authors solve a similar portfolio-consumption problem, but without model uncertainty, borrowing costs and consumption constraints. 


\section{Nonlinear ODE characterization of the value functions}

In this section, we apply a martingale argument, firstly introduced in [7] and [15], to construct a saddle point strategy $\left\{\left(\mu^{*}, \Sigma^{*}\right),\left(\pi^{*}, c^{*}\right)\right\}$ for the expected utility $\mathcal{J}_{i}(x ; \pi, c, \mu, \Sigma)$. This will in turn solve the original maxmin problem (5).

To this end, we aim to construct an $\mathbb{F}$-adapted process $J_{i, t}^{x ; \pi, c, \mu, \Sigma}, t \in[0, T]$, satisfying the following three conditions: For any $(\pi, c) \in \mathcal{A}$ and $(\mu, \Sigma) \in \mathcal{B}$,

(C1) at the maturity $T$,

$$
J_{i, T}^{x ; \pi, c, \mu, \Sigma}=\int_{0}^{T} \lambda e^{-\rho s} U_{i}^{c}\left(c_{S} X_{s}^{x ; \pi, c, \mu, \Sigma}\right) d s+e^{-\rho T} U_{i}\left(X_{T}^{x ; \pi, c, \mu, \Sigma}\right)
$$

(C2) at the initial time $0, J_{i, 0}^{x ; \pi, c, \mu, \Sigma}=J_{i, 0}^{x}$, which is a constant and is independent of $(\pi, c)$ and $(\mu, \Sigma)$;

(C3) there exist $\left(\pi^{*}, c^{*}\right) \in \mathcal{A}$ and $\left(\mu^{*}, \Sigma^{*}\right) \in \mathcal{B}$ such that the process $J_{i}^{x ; \pi^{*}, c^{*}, \mu^{*}, \Sigma^{*}}$ is a martingale, $J_{i}^{x ; \pi, c, \mu^{*}, \Sigma^{*}}$ is a supermartingale, and $J_{i}^{x ; \pi^{*}, c^{*}, \mu, \Sigma}$ is a submartingale.

Following the above conditions (C1-C3), we then have

$$
\begin{aligned}
\mathcal{J}_{i}\left(x ; \pi, c, \mu^{*}, \Sigma^{*}\right) & =E\left[J_{i, T}^{x ; \pi, c, \mu^{*}, \Sigma^{*}}\right] \leq J_{i, 0}^{x ; \pi, c, \mu^{*}, \Sigma^{*}}=J_{i, 0}^{x} ; \\
\mathcal{J}_{i}\left(x ; \pi^{*}, c^{*}, \mu^{*}, \Sigma^{*}\right) & =E\left[J_{i, T}^{x ; \pi^{*}, c^{*}, \mu^{*}, \Sigma^{*}}\right]=J_{i, 0}^{x ; \pi^{*}, c^{*}, \mu^{*}, \Sigma^{*}}=J_{i, 0}^{x} ; \\
\mathcal{J}_{i}\left(x ; \pi^{*}, c^{*}, \mu, \Sigma\right) & =E\left[J_{i, T}^{x ; \pi^{*}, c^{*}, \mu, \Sigma}\right] \geq J_{i, 0}^{x ; \pi^{*}, c^{*}, \mu, \Sigma}=J_{i, 0}^{x} .
\end{aligned}
$$

Thus, the inequalities in (6) hold, i.e., $\left\{\left(\pi^{*}, c^{*}\right),\left(\mu^{*}, \Sigma^{*}\right)\right\}$ is a saddle point strategy of the expected utility $\mathcal{J}_{i}(x ; \pi, c, \mu, \Sigma)$, and the value function of the maxmin problem (5) is given by $J_{i}(x)=J_{i, 0}^{x}$.

Next, we construct the process $\mathcal{J}_{i}^{x ; \pi, c, \mu, \Sigma}$. We start with the following lemma, which reduces the original maxmin problem (5), which is an infinite dimensional optimization problem, to a finite dimensional one. To facilitate our discussions below, we introduce two functions $F_{i}(\cdot ; \cdot, \cdot ; \cdot, \cdot), i=P, L$, which characterize the optimal portfolio-consumption and the worst-case parameters locally,

$$
\begin{aligned}
& F_{i}\left(x_{q} ; x_{\pi}, x_{c} ; x_{\mu}, x_{\Sigma}\right) \\
:= & \left\{\begin{array}{l}
\frac{p-1}{2} x_{\pi}^{\mathrm{T}} x_{\Sigma} x_{\pi}+\left[x_{\mu}^{\mathrm{T}} x_{\pi}+r\left(1-1_{d}^{\mathrm{T}} x_{\pi}\right)^{+}-R\left(1-1_{d}^{\mathrm{T}} x_{\pi}\right)^{-}\right]+\frac{\lambda}{p} e^{-x_{q}} x_{c}^{p}-x_{c}, \quad i=P ; \\
-\frac{1}{2} x_{\pi}^{\mathrm{T}} x_{\Sigma} x_{\pi}+\left[x_{\mu}^{\mathrm{T}} x_{\pi}+r\left(1-1_{d}^{\mathrm{T}} x_{\pi}\right)^{+}-R\left(1-1_{d}^{\mathrm{T}} x_{\pi}\right)^{-}\right]+\lambda e^{-x_{q}} \ln x_{c}-x_{c}, \quad i=L ;
\end{array}\right.
\end{aligned}
$$

for $x_{q} \in \mathbb{R},\left(x_{\pi}, x_{c}\right) \in \mathbb{A}$ and $\left(x_{\mu}, x_{\Sigma}\right) \in \mathbb{B}$. Recall that $\mathbb{A}$ is convex and closed, and $\mathbb{B}$ is convex and compact.

Lemma 3.1 For $i=P, L$, the function $F_{i}\left(x_{q} ; \cdot, \cdot ; \cdot, \cdot\right)$ admits the following properties.

(i) The function $F_{i}\left(x_{q} ; \cdot, \cdot ; \cdot, \cdot\right)$ admits at least one saddle point $\left(\widetilde{x}_{\pi}^{*}\left(x_{q}\right), \widetilde{x}_{c}^{*}\left(x_{q}\right) ; \widetilde{x}_{\mu}^{*}\left(x_{q}\right)\right.$, $\left.\tilde{x}_{\Sigma}^{*}\left(x_{q}\right)\right)$, i.e. for any $x_{q} \in \mathbb{R},\left(x_{\pi}, x_{c}\right) \in \mathbb{A}$ and $\left(x_{\mu}, x_{\Sigma}\right) \in \mathbb{B}$, 


$$
\begin{aligned}
F_{i}\left(x_{q} ; \tilde{x}_{\pi}^{*}\left(x_{q}\right), \tilde{x}_{c}^{*}\left(x_{q}\right) ; x_{\mu}, x_{\Sigma}\right) & \geq F_{i}\left(x_{q} ; \tilde{x}_{\pi}^{*}\left(x_{q}\right), \tilde{x}_{c}^{*}\left(x_{q}\right) ; \tilde{x}_{\mu}^{*}\left(x_{q}\right), \tilde{x}_{\Sigma}^{*}\left(x_{q}\right)\right) \\
& \geq F_{i}\left(x_{q} ; x_{\pi}, x_{c} ; \tilde{x}_{\mu}^{*}\left(x_{q}\right), \tilde{x}_{\Sigma}^{*}\left(x_{q}\right)\right) .
\end{aligned}
$$

(ii) For $x_{q} \in \mathbb{R}$, let

$$
G_{i}\left(x_{q}\right):=F_{i}\left(x_{q}, \tilde{x}_{\pi}^{*}\left(x_{q}\right), \tilde{x}_{c}^{*}\left(x_{q}\right) ; \tilde{x}_{\mu}^{*}\left(x_{q}\right), \tilde{x}_{\Sigma}^{*}\left(x_{q}\right)\right) .
$$

Then, $G_{i}\left(x_{q}\right), \tilde{x}_{\pi}^{*}\left(x_{q}\right), \tilde{x}_{c}^{*}\left(x_{q}\right), \tilde{x}_{\mu}^{*}\left(x_{q}\right)$ and $\tilde{x}_{\Sigma}^{*}\left(x_{q}\right)$ are locally bounded in $x_{q} \in \mathbb{R}$.

(iii) If $p<0$ or $i=L$, then $\left(\tilde{x}_{c}^{*}\left(x_{q}\right)\right)^{-1}$ is also locally bounded in $x_{q} \in \mathbb{R}$.

Proof Step 1 We first prove the assertion (i) when the set $\mathbb{A}$ is compact. Indeed, for fixed $x_{q} \in \mathbb{R}$, it is clear that the function $F_{i}\left(x_{q} ; \cdot, \cdot ; \cdot, \cdot\right)$ is concave with respect to $\left(x_{\pi}, x_{c}\right)$, and convex (accurately linear) with respect to $\left(x_{\mu}, x_{\Sigma}\right)$. Since $\mathbb{A}$ and $\mathbb{B}$ are convex and compact, we may apply the minmax theorem (see Theorem B on pp. 131 in [25] or Sect. 3 in [23]), and deduce that there exists a saddle point $\left(\widetilde{x}_{\pi}^{*}, \tilde{x}_{c}^{*} ; \tilde{x}_{\mu}^{*}, \tilde{x}_{\Sigma}^{*}\right)$ such that (9) holds. Moreover, the compactness of $\mathbb{A}$ and $\mathbb{B}$ implies that $\tilde{x}_{\pi}^{*}, \tilde{x}_{c}^{*}, \tilde{x}_{\mu}^{*}, \tilde{x}_{\Sigma}^{*}$ are bounded.

Step 2 If the set $\mathbb{A}$ is not compact, for any positive integer $n$, let $\mathbb{A}_{n}:=\mathbb{A} \cap\left\{\left(x_{\pi}, x_{c}\right)\right.$ : $\left.\left|\left(x_{\pi}, x_{c}\right)\right| \leq n\right\}$. It is clear that we can choose a large enough positive integer $N$ such that $\mathbb{A}_{n}$ is non-empty for any $n \geq N$ and, without loss of generality, we may suppose that $n \geq N$ below. Thanks to Step 1 , we know that the function $F_{i}\left(x_{q} ; \cdot, \cdot ; \cdot, \cdot\right)$ has at least one saddle point $\left(\widetilde{x}_{\pi}^{n}, \widetilde{x}_{c}^{n} ; \widetilde{x}_{\mu}^{n}, \widetilde{x}_{\Sigma}^{n}\right)$ in $\mathbb{A}_{n} \times \mathbb{B}$, and we denote $F_{i}\left(x_{q} ; \widetilde{x}_{\pi}^{n}, \widetilde{x}_{c}^{n} ; x_{\mu}^{n}, x_{\Sigma}^{n}\right)$ by $F_{i}^{n}$.

Next, we prove that $F_{i}^{n}$ is nondecreasing with respect to $n$ and has a uniformly lower bound for any $n \geq N$. To this end, note that

$$
\begin{aligned}
F_{i}^{n} & =\inf _{\left(x_{\mu}, x_{\Sigma}\right) \in \mathbb{B}} \sup _{\left(x_{\pi}, x_{c}\right) \in \mathbb{A}_{n}} F_{i}\left(x_{q} ; x_{\pi}, x_{c} ; x_{\mu}, x_{\Sigma}\right)=\sup _{\left(x_{\pi}, x_{c}\right) \in \mathbb{A}_{n}} \inf _{\left(x_{\mu}, x_{\Sigma}\right) \in \mathbb{B}} F_{i}\left(x_{q} ; x_{\pi}, x_{c} ; x_{\mu}, x_{\Sigma}\right) \\
& =\sup _{\left(x_{\pi}, x_{c}\right) \in \mathbb{A}_{n}} F_{i}\left(x_{q} ; x_{\pi}, x_{c} ; \tilde{x}_{\mu}^{n}, \widetilde{x}_{\Sigma}^{n}\right)=\inf _{\left(x_{\mu}, x_{\Sigma}\right) \in \mathbb{B}} F_{i}\left(x_{q} ; \widetilde{x}_{\pi}^{n}, \tilde{x}_{c}^{n} ; x_{\mu}, x_{\Sigma}\right) .
\end{aligned}
$$

From the first equality in (11), we deduce that $F_{i}^{n}$ is nondecreasing with respect to $n$. Furthermore, the second equality in (11) implies that, for any $n \geq N$ and $\left(x_{\pi}^{0}, x_{c}^{0}\right) \in \mathbb{A}_{N}$,

$$
F_{i}^{n} \geq F_{i}^{N} \geq \inf _{\left(x_{\mu}, x_{\Sigma}\right) \in \mathbb{B}} F_{i}\left(x_{q} ; x_{\pi}^{0}, x_{c}^{0} ; x_{\mu}, x_{\Sigma}\right)>-\infty,
$$

where we have used the fact that $\mathbb{B}$ is compact in the last inequality. Until now, we have proved that $F_{i}^{n}$ is nondecreasing with respect to $n$ and has a uniformly lower bound for any $n \geq N$.

Step 3 We prove that there exists a large enough positive integer $M$ such that $\left(\widetilde{x}_{\pi}^{n}, \widetilde{x}_{c}^{n}\right) \in \mathbb{A}_{M}$ for any $n \geq M$. Indeed, we may choose a positive constant $\epsilon$ and a positive-definite matrix $x_{\Sigma}^{0}$ such that $\left(x_{\mu}^{0}, x_{\Sigma}^{0}\right) \in \mathbb{B}$ and $x_{\pi}^{\mathrm{T}} x_{\Sigma}^{0} x_{\pi} \geq \epsilon\left|x_{\pi}\right|^{2}$ for any $x_{\pi} \in \mathbb{R}^{d}$. Hence, as $x_{c} \rightarrow 0^{+}$ when $p<0$ or $i=L$, or $\left|\left(x_{\pi}, x_{c}\right)\right| \rightarrow+\infty$, the compactness of $\mathbb{B}$ implies that

$$
\left\{\begin{array}{l}
F_{P}\left(x_{q} ; x_{\pi}, x_{c} ; x_{\mu}^{0}, x_{\Sigma}^{0}\right) \leq\left(\frac{p-1}{2} \epsilon\left|x_{\pi}\right|^{2}+C\left|x_{\pi}\right|\right)+\left(\frac{\lambda}{p} e^{-x_{q}} x_{c}^{p}-x_{c}\right) \rightarrow-\infty, \\
F_{L}\left(x_{q} ; x_{\pi}, x_{c} ; x_{\mu}^{0}, x_{\Sigma}^{0}\right) \leq\left(-\frac{\epsilon}{2}\left|x_{\pi}\right|^{2}+C\left|x_{\pi}\right|\right)+\left(\lambda e^{-x_{q}} \ln x_{c}-x_{c}\right) \rightarrow-\infty,
\end{array}\right.
$$

for any $\left(x_{\pi}, x_{c}\right) \in \mathbb{A}$, where $C$ is a constant independent of $x_{q}, x_{\pi}, x_{c}, x_{\mu}$ and $x_{\Sigma}$, In turn, there exists a large enough positive integer $M \geq N$ such that for any $\left(x_{\pi}, x_{c}\right) \in \mathbb{A} \backslash \mathbb{A}_{M}$, or for any $\left(x_{\pi}, x_{c}\right) \in \mathbb{A}$ with $x_{c}<1 / M$ when $p<0$ or $i=L$,

$\inf _{\left(x_{\mu}, x_{\Sigma}\right) \in \mathbb{B}} F_{i}\left(x_{q} ; x_{\pi}, x_{c} ; x_{\mu}, x_{\Sigma}\right) \leq F_{i}\left(x_{q} ; x_{\pi}, x_{c} ; x_{\mu}^{0}, x_{\Sigma}^{0}\right)<F_{i}^{N} \leq F_{i}^{M} \leq F_{i}^{n}, \quad n \geq M$. 
For the last two inequalities, we have used the fact that $F_{i}^{n}$ is nondecreasing with respect to $n$ (see Step 2). Thus, the last equality in (11) implies that $\left(\widetilde{x}_{\pi}^{n}, \widetilde{x}_{c}^{n}\right) \in \mathbb{A}_{M}$ for any $n \geq M$ and, moreover, $\tilde{x}_{c}^{n} \geq 1 / M$ when $p<0$ or $i=L$.

Step 4 We prove that the function $F_{i}\left(x_{q} ; \cdot, \cdot ; \cdot, \cdot\right)$ has at least one saddle point $\left(\tilde{x}_{\pi}^{*}, \tilde{x}_{c}^{*} ; \tilde{x}_{\mu}^{*}, \tilde{x}_{\Sigma}^{*}\right)$ in $\mathbb{A} \times \mathbb{B}$. Indeed, according to Step $3, F_{i}\left(x_{q} ; \cdot, \cdot ; \cdot, \cdot\right)$ has at least one saddle point $\left(\widetilde{x}_{\pi}^{n}, \widetilde{x}_{c}^{n} ; \widetilde{x}_{\mu}^{n}, \widetilde{x}_{\Sigma}^{n}\right)$ in $\mathbb{A}_{n} \times \mathbb{B}$, and all of them belong to a compact set $\mathbb{A}_{M} \times \mathbb{B}$ for any $n \geq M$. Hence, there exists a subsequence (still denoted by itself) such that $\left(\widetilde{x}_{\pi}^{n}, \widetilde{x}_{c}^{n} ; \tilde{x}_{\mu}^{n}, \widetilde{x}_{\Sigma}^{n}\right) \rightarrow\left(\tilde{x}_{\pi}^{*}, \tilde{x}_{c}^{*} ; \tilde{x}_{\mu}^{*}, \tilde{x}_{\Sigma}^{*}\right) \in \mathbb{A}_{M} \times \mathbb{B} \subseteq \mathbb{A} \times \mathbb{B}$. Next, we prove that $\left(\tilde{x}_{\pi}^{*}, \tilde{x}_{c}^{*} ; \tilde{x}_{\mu}^{*}, \tilde{x}_{\Sigma}^{*}\right)$ is a saddle point of $F_{i}\left(x_{q} ; \cdot, \cdot ; \cdot, \cdot\right)$ in $\mathbb{A} \times \mathbb{B}$.

It clear that

$$
F_{i}\left(x_{q} ; \tilde{x}_{\pi}^{n}, \widetilde{x}_{c}^{n} ; x_{\mu}, x_{\Sigma}\right) \geq F_{i}\left(x_{q} ; \widetilde{x}_{\pi}^{n}, \widetilde{x}_{c}^{n} ; \widetilde{x}_{\mu}^{n}, \widetilde{x}_{\Sigma}^{n}\right) \geq F_{i}\left(x_{q} ; x_{\pi}, x_{c} ; \widetilde{x}_{\mu}^{n}, \widetilde{x}_{\Sigma}^{n}\right),
$$

for any $\left(x_{\pi}, x_{c}\right) \in \mathbb{A}_{n}$ and $\left(x_{\mu}, x_{\Sigma}\right) \in \mathbb{B}$. Sending $n \rightarrow+\infty$ in the first inequality in (14), we deduce that for any $\left(x_{\mu}, x_{\Sigma}\right) \in \mathbb{B}$,

$$
F_{i}\left(x_{q} ; \tilde{x}_{\pi}^{*}, \tilde{x}_{c}^{*} ; x_{\mu}, x_{\Sigma}\right) \geq F_{i}\left(x_{q} ; \tilde{x}_{\pi}^{*}, \tilde{x}_{c}^{*} ; \tilde{x}_{\mu}^{*}, \tilde{x}_{\Sigma}^{*}\right) .
$$

On the other hand, for any $\left(x_{\pi}, x_{c}\right) \in \mathbb{A}$, we can choose a large enough positive integer $\tilde{N}$ such that $\left(x_{\pi}, x_{c}\right) \in \mathbb{A}_{n}$ for any $n \geq \tilde{N}$. Then, sending $n \rightarrow+\infty$ in the second inequality in (14), we deduce that

$$
F_{i}\left(x_{q} ; \tilde{x}_{\pi}^{*}, \tilde{x}_{c}^{*} ; \tilde{x}_{\mu}^{*}, \tilde{x}_{\Sigma}^{*}\right) \geq F_{i}\left(x_{q} ; x_{\pi}, x_{c} ; x_{\mu}^{*}, x_{\Sigma}^{*}\right) .
$$

Therefore, $\left(\tilde{x}_{\pi}^{*}, \tilde{x}_{c}^{*} ; \tilde{x}_{\mu}^{*}, \tilde{x}_{\Sigma}^{*}\right)$ is a saddle point of $F_{i}\left(x_{q} ; \cdot, \cdot ; \cdot, \cdot\right)$ in $\mathbb{A} \times \mathbb{B}$.

Step 5 We prove that assertions (ii) and (iii) hold. Indeed, from the proof in Step 4, we know that all saddle points $\left(\widetilde{x}_{\pi}^{n}, \widetilde{x}_{c}^{n} ; \widetilde{x}_{\mu}^{n}, \widetilde{x}_{\Sigma}^{n}\right)$ belong to a compact set $\mathbb{A}_{M} \times \mathbb{B}$ for any $n \geq M$ and $x_{q} \in \mathbb{R}$. Furthermore, it follows from (12) and (13) in Step 3 that, there exists a neighborhood of $x_{q}$, say $x_{q} \in(a, b)$, such that the subscript $M$ in $\mathbb{A}_{M}$ is independent of $x_{q}$ (but may depend on $a$ and $b)$. Thus, for $x_{q} \in(a, b),\left(\tilde{x}_{\pi}^{*}\left(x_{q}\right), \widetilde{x}_{c}^{*}\left(x_{q}\right), \tilde{x}_{\mu}^{*}\left(x_{q}\right), \tilde{x}_{\Sigma}^{*}\left(x_{q}\right)\right) \in \mathbb{A}_{M} \times \mathbb{B}$ which means the functions $\tilde{x}_{\pi}^{*}\left(x_{q}\right), \tilde{x}_{c}^{*}\left(x_{q}\right), \tilde{x}_{\mu}^{*}\left(x_{q}\right)$ and $\tilde{x}_{\Sigma}^{*}\left(x_{q}\right)$ are locally bounded and, moreover, (8) and (10) imply that $G_{i}\left(x_{q}\right)$ is also locally bounded in $x_{q} \in \mathbb{R}$.

From Step 3, we know that for any $n \geq M, \widetilde{x}_{c}^{n} \geq 1 / M$ when $p<0$ or $i=L$. Since the saddle point $\left(\widetilde{x}_{\pi}^{*}, \widetilde{x}_{c}^{*} ; \widetilde{x}_{\mu}^{*}, \widetilde{x}_{\Sigma}^{*}\right)$ is the limit of $\left(\widetilde{x}_{\pi}^{n}, \widetilde{x}_{c}^{n} ; \widetilde{x}_{\mu}^{n}, \widetilde{x}_{\Sigma}^{n}\right)$, we deduce that $\widetilde{x}_{c}^{*} \geq 1 / M$ in the case of $p<0$ or $i=L$, which means that $\left(\tilde{x}_{c}^{*}\left(x_{q}\right)\right)^{-1}$ is locally bounded in $x_{q} \in \mathbb{R}$.

We are now ready to state our first main result, which is about nonlinear ODE characterization of the value functions $J_{i}(\cdot)$ for $i=P, L$. Since the conclusions for power and logarithm utility functions are different, we present their results separately.

Theorem 3.2 Suppose that $q_{P}(\cdot)$ solves the following nonlinear ODE

$$
q_{P}(t)=\int_{t}^{T}\left[p G_{P}\left(q_{P}(s)\right)-\rho\right] d s, \quad t \in[0, T],
$$

where the function $G_{P}(\cdot)$ is given in Lemma 3.1.

Then, for the power utility case, the process

$$
J_{P, t}^{x ; \pi, c, \mu, \Sigma}:=\frac{1}{p} \int_{0}^{t} \lambda e^{-\rho s}\left(c_{s} X_{s}^{x ; \pi, c, \mu, \Sigma}\right)^{p} d s+\frac{1}{p} e^{q_{P}(t)-\rho t}\left(X_{t}^{x ; \pi, c, \mu, \Sigma}\right)^{p},
$$

together with $\left(\pi_{t}^{*}, c_{t}^{*}\right)=\left(\tilde{x}_{\pi}^{*}\left(q_{P}(t)\right), \tilde{x}_{c}^{*}\left(q_{P}(t)\right)\right)$ and $\left(\mu_{t}^{*}, \Sigma_{t}^{*}\left(\Sigma_{t}^{*}\right)^{T}\right)=\left(\widetilde{x}_{\mu}^{*}\left(q_{P}(t)\right), \tilde{x}_{\Sigma}^{*}\right.$ $\left.\left(q_{P}(t)\right)\right), t \in[0, T]$, satisfy the conditions $(C 1-C 3)$, where $\left(\tilde{x}_{\pi}^{*}\left(x_{q}\right), \tilde{x}_{c}^{*}\left(x_{q}\right) ; \tilde{x}_{\mu}^{*}\left(x_{q}\right), \tilde{x}_{\Sigma}^{*}\left(x_{q}\right)\right)$ 
is a saddle point given in Lemma 3.1. In particular, the value function of the maximin problem (5) is given by

$$
J_{P}(x)=J_{P, 0}^{x}=\frac{x^{p}}{p} e^{q_{P}(0)}
$$

Proof $J_{P}^{x ; \pi, c, \mu, \Sigma}$ in (16) obviously satisfies the conditions (C1) and (C2), so it suffices to verify the martingale property $(\mathrm{C} 3)$.

To this end, for any $(\pi, c) \in \mathcal{A}$ and $(\mu, \Sigma) \in \mathcal{B}$, an application of Itô's formula implies

$$
\begin{aligned}
d\left(X_{s}^{x ; \pi, c, \mu, \Sigma}\right)^{p}= & \left(X_{s}^{x ; \pi, c, \mu, \Sigma}\right)^{p}\left\{\left[p F_{P}\left(q_{P}(s) ; \pi_{s}, c_{s} ; \mu_{s}, \Sigma_{s} \Sigma_{s}^{\mathrm{T}}\right)-\lambda e^{-q_{P}(s)} c_{s}^{p}\right] d s\right. \\
& \left.+p \pi_{s}^{\mathrm{T}} \Sigma_{s} d W_{s}\right\}
\end{aligned}
$$

and in turn,

$$
\begin{aligned}
J_{P, t}^{x ; \pi, c, \mu, \Sigma}= & J_{P, 0}^{x ; \pi, c, \mu, \Sigma} \\
& +\int_{0}^{t} e^{q_{P}(s)-\rho s}\left(X_{s}^{x ; \pi, c, \mu, \Sigma}\right)^{p}\left[F_{P}\left(q_{P}(s) ; \pi_{s}, c_{s} ; \mu_{s}, \Sigma_{s} \Sigma_{s}^{\mathrm{T}}\right)+\frac{q_{P}^{\prime}(s)-\rho}{p}\right] d s \\
& +\int_{0}^{t} e^{q_{P}(s)-\rho s}\left(X_{s}^{x ; \pi, c, \mu, \Sigma}\right)^{p} \pi_{s}^{\mathrm{T}} \Sigma_{s} d W_{s} .
\end{aligned}
$$

Since $q_{P}(\cdot)$ is a continuous and deterministic function, we know that $q_{P}$ is bounded in the interval $[0, T]$. Together with Lemma 3.1, we deduce that $G_{P}\left(q_{P}(\cdot)\right)$ and $\pi^{*}, c^{*}, \mu, \Sigma^{*}$ are all bounded, and $\left(c^{*}\right)^{-1}$ is also bounded when $p<0$. It follows that the stochastic exponential $\mathcal{E}\left(p \int_{0}^{*}\left(\pi_{s}^{*}\right)^{\mathrm{T}} \Sigma_{s}^{*} d W_{s}\right)$ is a uniformly integrable martingale. Moreover, from (17), we deduce that

$$
\begin{aligned}
& \left(X_{t}^{x, \pi^{*}, c^{*}, \mu^{*}, \Sigma^{*}}\right)^{p} \\
& =x^{p} \mathcal{E}_{t}\left(p \int_{0}^{\cdot}\left(\pi_{s}^{*}\right)^{\mathrm{T}} \Sigma_{s}^{*} d W_{s}\right) \exp \left(\int_{0}^{t}\left[p G_{P}\left(q_{P}(s)\right)-\lambda e^{-q_{P}(s)}\left(c_{s}^{*}\right)^{p}\right] d s\right)
\end{aligned}
$$

for $t \in[0, T]$. Moreover, there exists a constant $C>0$ such that

$$
E\left[\int_{0}^{T}\left(c_{t}^{*} X_{t}^{x, \pi^{*}, c^{*}, \mu^{*}, \Sigma^{*}}\right)^{p} d t\right] \leq C E\left[\int_{0}^{T} \mathcal{E}_{t}\left(p \int_{0}^{\cdot}\left(\pi_{s}^{*}\right)^{\mathrm{T}} \Sigma_{s}^{*} d W_{s}\right) d t\right]=C T .
$$

Thus, $X^{x, \pi^{*}, c^{*}, \mu^{*}, \Sigma^{*}}$ satisfies the condition $(\mathrm{H})$, and $\left(\pi^{*}, c^{*}\right) \in \mathcal{A}$ and $\left(\mu^{*}, \Sigma^{*}\right) \in \mathcal{B}$. Together with ODE (15) for $q_{P}(\cdot)$, we deduce that

$$
E\left[J_{P, s}^{x ; \pi^{*}, c^{*}, \mu^{*}, \Sigma^{*}} \mid \mathcal{F}_{t}\right]=J_{P, t}^{x ; \pi^{*}, c^{*}, \mu^{*}, \Sigma^{*}}
$$

for any $0 \leq t \leq s \leq T$.

With $\left(\mu_{s}^{*}, \Sigma_{s}^{*}\left(\Sigma_{s}^{*}\right)^{T}\right)=\left(\widetilde{x}_{\mu}^{*}\left(q_{P}(s)\right), \widetilde{x}_{\Sigma}^{*}\left(q_{P}(s)\right)\right)$, the second inequality in the saddle point condition (9) implies

$$
F_{P}\left(q_{P}(s) ; \pi_{s}, c_{s} ; \mu_{s}^{*}, \Sigma_{s}^{*}\left(\Sigma_{s}^{*}\right)^{\mathrm{T}}\right)+\frac{q_{P}^{\prime}(s)-\rho}{p} \leq G_{P}\left(q_{P}(s)\right)+\frac{q_{P}^{\prime}(s)-\rho}{p}=0
$$

for any $(\pi, c) \in \mathcal{A}$. Thus $J_{P}^{x ; \pi, c, \mu^{*}, \Sigma^{*}}$ is a local supermartingale. Take an increasing sequence of $\mathbb{F}$-stopping times $\tau_{n} \uparrow T$ such that for any $0 \leq t \leq s \leq T$, 


$$
E\left[J_{P, s \wedge \tau_{n}}^{x ; \pi, c, \mu^{*}, \Sigma^{*}} \mid \mathcal{F}_{t}\right] \leq J_{P, t \wedge \tau_{n}}^{x ; \pi, c, \mu^{*}, \Sigma^{*}}
$$

i.e.

$$
E\left[J_{P, s \wedge \tau_{n}}^{x ; \pi, c, \mu^{*}, \Sigma^{*}} 1_{A}\right] \leq E\left[J_{P, t \wedge \tau_{n}}^{x ; \pi, \mu^{*}, \Sigma^{*}} 1_{A}\right]
$$

for any $A \in \mathcal{F}_{t}$. By the condition (H) on $X^{x, \pi, c, \mu^{*}, \Sigma^{*}}$, we may let $\tau_{n} \uparrow T$ in (18), which then implies that $E\left[J_{P, s}^{x ; \pi, c, \mu^{*}, \Sigma^{*}} 1_{A}\right] \leq E\left[J_{P, t}^{x ; \pi, c, \mu^{*}, \Sigma^{*}} 1_{A}\right]$, i.e. $J_{P}^{x ; \pi, c, \mu^{*}, \Sigma^{*}}$ is a supermartingale.

Finally, with $\left(\pi_{s}^{*}, c_{s}^{*}\right)=\left(\widetilde{x}_{\pi}^{*}\left(q_{P}(s)\right), \widetilde{x}_{c}^{*}\left(q_{P}(s)\right)\right)$, the first inequality in the saddle point condition (9) implies

$$
F_{P}\left(q_{P}(s) ; \pi_{s}^{*}, c_{s}^{*} ; \mu_{s}, \Sigma_{s} \Sigma_{s}^{\mathrm{T}}\right)+\frac{q_{P}^{\prime}(s)-\rho}{p} \geq G_{P}\left(q_{P}(s)\right)+\frac{q_{P}^{\prime}(s)-\rho}{p}=0
$$

for any $(\mu, \Sigma) \in \mathcal{B}$, so $J_{P}^{x ; \pi^{*}, c^{*}, \mu, \Sigma}$ is a local submartingale. Following along similar argument as above, we obtain that $J_{P}^{x ; \pi^{*}, c^{*}, \mu, \Sigma}$ is a submartingale.

Theorem 3.3 Suppose that $q_{L}(\cdot)$ and $Q_{L}(\cdot)$ solve the following ODEs

$$
q_{L}(t)=\int_{t}^{T}\left(\lambda e^{-q_{L}(s)}-\rho\right) d s, \quad Q_{L}(t)=\int_{t}^{T} e^{q_{L}(s)-\rho s} G_{L}\left(q_{L}(s)\right) d s, \quad t \in[0, T],
$$

where the function $G_{L}(\cdot)$ is given in Lemma 3.1.

Then, for the logarithm utility case, the process

$$
J_{L, t}^{x ; \pi, c, \mu, \Sigma}:=\int_{0}^{t} \lambda e^{-\rho s} \ln \left(c_{s} X_{s}^{x ; \pi, c, \mu, \Sigma}\right) d s+e^{q_{L}(t)-\rho t} \ln \left(X_{t}^{x ; \pi, c, \mu, \Sigma}\right)+Q_{L}(t),
$$

together with $\left(\pi_{t}^{*}, c_{t}^{*}\right)=\left(\tilde{x}_{\pi}^{*}\left(q_{L}(t)\right), \tilde{x}_{c}^{*}\left(q_{L}(t)\right)\right)$ and $\left(\mu_{t}^{*}, \Sigma_{t}^{*}\left(\Sigma_{t}^{*}\right)^{T}\right)=\left(\tilde{x}_{\mu}^{*}\left(q_{L}(t)\right), \tilde{x}_{\Sigma}^{*}\right.$ $\left.\left(q_{L}(t)\right)\right), t \in[0, T]$, satisfy the conditions $(C 1-C 3)$, where $\left(\widetilde{x}_{\pi}^{*}\left(x_{q}\right), \widetilde{x}_{c}^{*}\left(x_{q}\right) ; \tilde{x}_{\mu}^{*}\left(x_{q}\right), \tilde{x}_{\Sigma}^{*}\left(x_{q}\right)\right)$ is a saddle point given in Lemma 3.1. In particular, the value function of the maximin problem (5) is given by

$$
J_{L}(x)=J_{L, 0}^{x}=e^{q_{L}(0)} \ln x+Q_{L}(0) .
$$

Proof $J_{L}^{x ; \pi, c, \mu, \Sigma}$ in (20) obviously satisfies the conditions (C1) and (C2), so it suffices to verify the martingale property $(\mathrm{C} 3)$.

To this end, for any $(\pi, c) \in \mathcal{A}$ and $(\mu, \Sigma) \in \mathcal{B}$, an application of Itô's formula implies

$$
d \ln \left(X_{s}^{x ; \pi, c, \mu, \Sigma}\right)=\left[F_{L}\left(q_{L}(s) ; \pi_{s}, c_{s} ; \mu_{s}, \Sigma_{s} \Sigma_{s}^{\mathrm{T}}\right)-\lambda e^{-q_{L}(s)} \ln c_{s}\right] d s+\pi_{s}^{\mathrm{T}} \Sigma_{s} d W_{s},
$$

and in turn,

$$
\begin{aligned}
J_{L, t}^{x ; \pi, c, \mu, \Sigma}= & J_{L, 0}^{x ; \pi, c, \mu, \Sigma} \\
& +\int_{0}^{t} e^{q_{L}(s)-\rho s}\left\{\left[F_{L}\left(q_{L}(s) ; \pi_{s}, c_{s} ; \mu_{s}, \Sigma_{s} \Sigma_{s}^{\mathrm{T}}\right)+e^{-q_{L}(s)+\rho s} Q_{L}^{\prime}(s)\right]\right. \\
& \left.+\left[q_{L}^{\prime}(s)-\rho+\lambda e^{-q_{L}(s)}\right] \ln X_{s}^{x ; \pi, c, \mu, \Sigma}\right\} d s+\int_{0}^{t} e^{q_{L}(s)-\rho s} \pi_{s}^{\mathrm{T}} \Sigma_{s} d W_{s}
\end{aligned}
$$




$$
\begin{aligned}
= & J_{L, 0}^{x ; \pi, c, \mu, \Sigma}+\int_{0}^{t}\left[e^{q_{L}(s)-\rho s} F_{L}\left(q_{L}(s) ; \pi_{s}, c_{s} ; \mu_{s}, \Sigma_{s} \Sigma_{s}^{\mathrm{T}}\right)+Q_{L}^{\prime}(s)\right] d s \\
& +\int_{0}^{t} e^{q_{L}(s)-\rho s} \pi_{s}^{\mathrm{T}} \Sigma_{s} d W_{s} .
\end{aligned}
$$

Since $q_{L}(\cdot), Q_{L}(\cdot)$ are continuous and deterministic functions, we know that $q_{L}(\cdot), Q_{L}(\cdot)$ are bounded in the interval $[0, T]$. Together with Lemma 3.1, we deduce that $G_{L}\left(q_{L}(\cdot)\right)$ and $\pi^{*}, c^{*}, \mu, \Sigma^{*},\left(c^{*}\right)^{-1}$ are all bounded. It follows that the stochastic integral $\int_{0}^{*}\left(\pi_{s}^{*}\right)^{\mathrm{T}} \Sigma_{s}^{*} d W_{s}$ is a uniformly integrable martingale. Moreover, from (21), we deduce that

$$
\ln \left(X_{t}^{x, \pi^{*}, c^{*}, \mu^{*}, \Sigma^{*}}\right)=\ln x+\int_{0}^{t}\left[G_{L}\left(q_{L}(s)\right)-\lambda e^{-q_{L}(s)} \ln c_{s}\right] d s+\int_{0}^{t} \pi_{s}^{\mathrm{T}} \Sigma_{s} d W_{s}
$$

for $t \in[0, T]$. Since $\left|\ln c^{*}\right| \leq c^{*}+\left(c^{*}\right)^{-1}$, and there exists a constant $C>0$ such that

$$
\begin{aligned}
E\left[\int_{0}^{T}\left|\ln \left(c_{s}^{*} X_{s}^{x, \pi^{*}, c^{*}, \mu^{*}, \Sigma^{*}}\right)\right| d s\right] \leq & E\left[\int_{0}^{T}\left|\ln c_{s}^{*}\right| d s\right] \\
& +E\left[\int_{0}^{T}\left|\ln X_{s}^{x, \pi^{*}, c^{*}, \mu^{*}, \Sigma^{*}}\right| d s\right] \leq C T,
\end{aligned}
$$

we deduce that $X^{x, \pi, c, \mu^{*}, \Sigma^{*}}$ satisfies the condition $(\mathrm{H})$, and $\left(\pi^{*}, c^{*}\right) \in \mathcal{A}$ and $\left(\mu^{*}, \Sigma^{*}\right) \in \mathcal{B}$. Together with the two ODEs (19) for $q_{L}(\cdot)$ and $Q_{L}(\cdot)$, we obtain

$$
E\left[J_{L, s}^{x ; \pi^{*}, c^{*}, \mu^{*}, \Sigma^{*}} \mid \mathcal{F}_{t}\right]=J_{L, t}^{x ; \pi^{*}, c^{*}, \mu^{*}, \Sigma^{*}}
$$

for any $0 \leq t \leq s \leq T$. The rest of the proof is similar to that of Theorem 3.2 and is thus omitted.

\subsection{One-dimensional case}

In the rest of this paper, we focus on one-dimensional case, and derive explicit solutions for the optimal investment-consumption strategies and the worst-case parameters. Assume that $d=d^{\prime}=1$ and $\mathbb{A}=[\underline{\pi}, \bar{\pi}] \times[\underline{c}, \bar{c}]$, where $\underline{\pi}, \bar{\pi}, \underline{c}, \bar{c}$ are constants satisfying $-\infty \leq \underline{\pi} \leq$ $0,1 \leq \bar{\pi} \leq+\infty, 0 \leq \underline{c} \leq \bar{c} \leq+\infty$.

For $i=P, L$, we split the function $F_{i}$ [cf. (8)] into two parts as $F_{i}\left(x_{q} ; x_{\pi}, x_{c} ; x_{\mu}, x_{\sigma}\right)=$ $f_{i}\left(x_{q} ; x_{c}\right)+g\left(x_{\pi} ; x_{\mu}, x_{\sigma}\right)$, where we used the notation $x_{\sigma}$ to replace $x_{\Sigma}$ in (8) to emphasize the one-dimensional setting, and $f_{i}, g$ are defined as follows,

$$
f_{i}\left(x_{q} ; x_{c}\right):=\left\{\begin{array}{l}
\frac{\lambda}{p} e^{-x_{q}} x_{c}^{p}-x_{c}, \quad i=P \\
\lambda e^{-x_{q}} \ln x_{c}-x_{c}, i=L
\end{array}\right.
$$

and

$$
g\left(x_{\pi} ; x_{\mu}, x_{\sigma}\right):=\frac{p-1}{2} x_{\sigma} x_{\pi}^{2}+\left(x_{\mu} x_{\pi}+r\left(1-x_{\pi}\right)-(R-r)\left(1-x_{\pi}\right)^{-}\right) .
$$

Herein, with a slight abuse of notation, we take $p=0$ in the function $g$ for $i=L$.

It is clear that for any $x_{q} \in \mathbb{R},\left(x_{\pi}^{*}, \tilde{x}_{c, i}^{*}\left(x_{q}\right) ; x_{\mu}^{*}, x_{\sigma}^{*}\right)$ is a saddle point of $F_{i}$ in $\mathbb{A} \times \mathbb{B}$, if $\tilde{x}_{c, i}^{*}\left(x_{q}\right)$ is the maximum point of $f_{i}\left(x_{q} ; \cdot\right)$ in the interval $[\underline{c}, \bar{c}]$ and $\left(x_{\pi}^{*} ; x_{\mu}^{*}, x_{\sigma}^{*}\right)$ is a saddle point of $g$ in $[\underline{\pi}, \bar{\pi}] \times \mathbb{B}$, i.e.

$$
f_{i}\left(x_{q} ; \tilde{x}_{c, i}^{*}\left(x_{q}\right)\right)=\max _{x_{c} \in[\underline{c}, \bar{c}]} f_{i}\left(x_{q} ; x_{c}\right) ;
$$




$$
g\left(x_{\pi} ; x_{\mu}^{*}, x_{\sigma}^{*}\right) \leq g\left(x_{\pi}^{*} ; x_{\mu}^{*}, x_{\sigma}^{*}\right) \leq g\left(x_{\pi}^{*} ; x_{\mu}, x_{\sigma}\right)
$$

for any $\left(x_{\pi} ; x_{\mu}, x_{\sigma}\right) \in[\underline{\pi}, \bar{\pi}] \times \mathbb{B}$.

From (22), it is immediate that the maximum value and maximum point of $f_{i}$ in the interval $[\underline{c}, \bar{c}]$ take the form

$$
\begin{aligned}
& f_{P}\left(x_{q} ; \tilde{x}_{c, P}^{*}\left(x_{q}\right)\right) \\
& \quad= \begin{cases}\frac{\lambda}{p} \bar{c}^{p} e^{-x_{q}}-\bar{c}, & \text { if } x_{q}<(p-1) \ln \bar{c}+\ln \lambda ; \\
\frac{(1-p) \lambda^{1 /(1-p)}}{p} e^{x_{q} /(p-1)}, & \text { if }(p-1) \ln \bar{c}+\ln \lambda \leq x_{q} \leq(p-1) \ln \underline{c}+\ln \lambda ; \\
\frac{\lambda}{p} \underline{c}^{p} e^{-x_{q}}-\underline{c}, & \text { if } x_{q}>(p-1) \ln \underline{c}+\ln \lambda .\end{cases}
\end{aligned}
$$

where

$$
\begin{aligned}
\tilde{x}_{c, P}^{*}\left(x_{q}\right) & :=\underline{c} 1_{\left\{\widehat{c}_{P}\left(x_{q}\right) \leq \underline{c}\right\}}+\widehat{c}_{P}\left(x_{q}\right) 1_{\left\{\underline{c}<\widehat{c}_{P}\left(x_{q}\right)<\bar{c}\right\}}+\bar{c} 1_{\left\{\widehat{c}_{P}\left(x_{q}\right) \geq \bar{c}\right\}}, \\
\widehat{c}_{P}\left(x_{q}\right) & :=\lambda^{\frac{1}{1-p}} \exp \left(\frac{x_{q}}{p-1}\right),
\end{aligned}
$$

and

$$
f_{L}\left(x_{q} ; \tilde{x}_{c, L}^{*}\left(x_{q}\right)\right)= \begin{cases}\lambda e^{-x_{q}} \ln \bar{c}-\bar{c}, & \text { if } x_{q}<\ln \lambda-\ln \bar{c} \\ \lambda e^{-x_{q}}\left(\ln \lambda-x_{q}-1\right), & \text { if } \ln \lambda-\ln \bar{c} \leq x_{q} \leq \ln \lambda-\ln \underline{c} ; \\ \lambda e^{-x_{q}} \ln \underline{c}-\underline{c}, & \text { if } x_{q}>\ln \lambda-\ln \underline{c} .\end{cases}
$$

where

$$
\widetilde{x}_{c, L}^{*}\left(x_{q}\right)=\underline{c} 1_{\left\{\widehat{c}_{L}\left(x_{q}\right) \leq \underline{c}\right\}}+\widehat{c}_{L}\left(x_{q}\right) 1_{\left\{\underline{c}<\widehat{c}_{L}\left(x_{q}\right)<\bar{c}\right\}}+\bar{c} 1_{\left\{\widehat{c}_{L}\left(x_{q}\right) \geq \bar{c}\right\}}, \widehat{c}_{L}\left(x_{q}\right):=\lambda e^{-x_{q}} .
$$

In the case of power utility function, the corresponding ODE (15) has a financial interpretation. The exponential of the ODE's solution $e^{q_{P}(t)}$ represents the investor's extra utilities obtained by optimizing over all admissible portfolio-consumption strategies (least affected by model uncertainty) in the remaining horizon $[t, T]$, and in the literature, $e^{q_{P}(t)}$ is dubbed as a (deterministic) opportunity process (see [24]).

Moreover, ODE (15) and the definition of $f_{P}(\cdot ; \cdot)$ imply

$$
\frac{-\left(e^{q_{P}(t)}\right)^{\prime}}{e^{q_{P}(t)}}=-q_{P}^{\prime}(t)=p f_{P}\left(q_{P}(t) ; c^{*}(t)\right)+p g\left(x_{\pi}^{*} ; x_{\mu}^{*}, x_{\sigma}^{*}\right)-\rho,
$$

where, with a slight abuse of notation, we denote

$$
c^{*}(t):=\tilde{x}_{c, P}^{*}\left(q_{P}(t)\right) .
$$

Hence, we can further interpret ODE (15) as a description of the relative changing rate of the opportunity process $e^{q_{P}(t)}$, which consists of three factors: (i) the consumption contributing factor $p f_{P}\left(q_{P}(\cdot), c^{*}(\cdot)\right)$, representing the change of the opportunity process due to the consumption optimization, and including two parts: current contribution $\lambda e^{-q_{P}(\cdot)}\left(c^{*}(\cdot)\right)^{p} / p$ and future contribution $-c^{*}(\cdot)$; (ii) the future investment contributing factor $p g\left(x_{\pi}^{*} ; x_{\mu}^{*}, x_{\sigma}^{*}\right)$, representing the change of the opportunity process due to the portfolio optimization in the remaining horizon; and (iii) the discount rate $\rho$. Increasing the consumption and future investment contributing factors or decreasing the discount rate will lead to a larger opportunity process.

The current consumption contributing factor is the only one affecting the instantanous utility, which is also reflected in the expression of the expected utility (4). The future consumption contributing factor and the future investment contributing factor determine the future consumption and terminal utility through the channel of the future wealth. The player 
achieves the maximum utility through balancing the risky asset and riskless asset via the investment strategy, while balancing the current utility and future utility via the consumption strategy. Moreover, the definition of $f_{P}\left(q_{P}(\cdot), c^{*}(\cdot)\right)$ implies that $\lambda e^{-q_{P}(\cdot)}$ is the weight of the current consumption utility relative to the future utility, which is consistent with our intuition that increasing opportunity process will lead to a larger weight of the future utility, and decrease the current consumption.

\section{Explicit solutions of the optimal strategies and worst-case parameters}

\subsection{The worst-case parameters and the optimal portfolios}

In this section, we further compute the saddle point $\left(x_{\pi}^{*} ; x_{\mu}^{*}, x_{\sigma}^{*}\right)$ of the function $g(\cdot ; \cdot, \cdot)$ given in (23). It then follows from Theorems 3.2 and 3.3 that the saddle point provides an explicit solution for the worst-case parameters and the optimal portfolio of the maxmin problem (5) by letting $\left(\mu_{s}^{*}, \sigma_{s}^{*}\right)=\left(x_{\mu}^{*}, \sqrt{x_{\sigma}^{*}}\right)$ and $\pi_{s}^{*}=x_{\pi}^{*}$ for $s \in[0, T]$. In particular, we consider two specific examples of the uncertain parameter sets $\mathbb{B}$.

Assumption 4.1 Assume that $\mathbb{B}=[\underline{\mu}, \bar{\mu}] \times\left[\underline{\sigma}^{2}, \bar{\sigma}^{2}\right]$, where $\underline{\mu}, \bar{\mu}, \underline{\sigma}, \bar{\sigma}$ are constants satisfying $-\infty<\underline{\mu} \leq \bar{\mu}<+\infty, 0 \leq \underline{\sigma} \leq \bar{\sigma}<+\infty$ and $\bar{\sigma}>0$.

Theorem 4.2 Under Assumption 4.1, the worst-case parameters $\left(\mu^{*}, \sigma^{*}\right)$ and the optimal portfolio $\pi^{*}$ are given as follows:

(i) The worst-case drift and volatility are

$$
\left(\mu_{s}^{*}, \sigma_{s}^{*}\right)=\left(\underline{\mu} 1_{\{\underline{\mu>r\}}}+[\underline{\mu}, \bar{\mu}] 1_{\{\underline{\mu} \leq r \leq \bar{\mu}\}}+\bar{\mu} 1_{\{\bar{\mu}<r\}}, \bar{\sigma}\right)
$$

for $s \in[0, T]$, where $[\mu, \bar{\mu}]$ means $\mu_{s}^{*}$ may take any value in that interval;

(ii) The optimal portfolio is a constant process, which is summarized in Table 1, with $\beta_{1}$, $\beta_{2}$ and $\beta_{3}$ given as

$$
\beta^{1}:=\frac{\underline{\mu}-R}{(1-p) \bar{\sigma}^{2}}, \quad \beta^{2}:=\frac{\underline{\mu}-r}{(1-p) \bar{\sigma}^{2}}, \quad \beta^{3}:=\frac{\bar{\mu}-r}{(1-p) \bar{\sigma}^{2}} .
$$

Proof Due to its length, the proof is postponed to "Appendix A".

We note that the worst-case volatility $\sigma^{*}$ attains its upper bound $\bar{\sigma}$. This is due to the fact that the value function is monotone in volatility $\sigma$ in the one-dimensional setting. A larger $\sigma$ means the investor faces more market risks, and therefore, she will have a smaller value function.

On the other hand, the worst-case drift is a bang-bang type. By the assertion (ii) about the optimal portfolio strategies, we know that $\mu>r$ implies $\pi^{*}>0$, i.e. the investor holds a long position of the stock. The worst-case drift is therefore its lower bound. Likewise, $\bar{\mu}<r$ implies $\pi^{*}<0$, and therefore, the worst-case drift takes its upper bound. If $\underline{\mu} \leq r \leq \bar{\mu}$, then $\pi^{*} \equiv 0$, so the estimation of the drift is irrelevant in this situation.

From Table 1, we categorize five different optimal portfolio strategies $\pi^{*}$ according to various scenarios.

(i) Borrow-to-buy strategy When $\beta^{1} \geq 1$, the investor will borrow $\left(\min \left\{\beta^{1}, \bar{\pi}\right\}-1\right)$ units of her wealth with borrowing rate $R$ to invest in the stock, and the optimal portfolio is $\min \left\{\beta^{1}, \bar{\pi}\right\}$. The reason is that in this situation, $\underline{\mu} \geq R+(1-p) \bar{\sigma}^{2}$, i.e. the stock's return 
Table 1 The optimal portfolio strategies

\begin{tabular}{llllll}
\hline & $\beta^{1} \geq 1$ & $\beta^{1} \leq 1 \leq \beta^{2}$ & $0 \leq \beta^{2} \leq 1$ & $\beta^{2} \leq 0 \leq \beta^{3}$ & $\beta^{3} \leq 0$ \\
\hline$\pi_{s}^{*}$ & $\min \left\{\beta^{1}, \bar{\pi}\right\}$ & 1 & $\beta^{2}$ & 0 & $\max \left\{\beta^{3}, \underline{\pi}\right\}$ \\
\hline
\end{tabular}

even with the worst estimation of the drift is still higher than the borrowing cost. Hence, the stock's high risk premium attracts the investor to borrow to invest as much as possible to approach the optimal strategy without constraint.

(ii) Full-position strategy When $\beta^{1} \leq 1 \leq \beta^{2}$, the investor will simply invest all her wealth in the stock with no additional borrowing or lending. In this case, since $\mu \leq R+(1-p) \bar{\sigma}^{2}$, there exists a possibility that the stock's return may not be good enough to compensate for the borrowing cost. As a result, the investor would prefer not to borrow. On the other hand, since $\mu \geq r+(1-p) \bar{\sigma}^{2}$, the stock's return even in the worst scenario is still better than the return from the bank account, and accordingly, the investor would put all her money in the stock rather than in the bank account.

(iii) Lend-and-buy strategy When $0 \leq \beta^{2} \leq 1$, the investor will invest $\beta^{2}$ proportion of her wealth in the stock, and the remaining proportion $\left(1-\beta^{2}\right)$ in the bank account to earn the interest rate $r$. This is similar to the standard Merton's strategy with Sharpe ratio $(\underline{\mu}-r) / \bar{\sigma}$.

(iv) No-trading strategy When $\beta^{2} \leq 0 \leq \beta^{3}$, the investor will put all her money in the bank account. In this case, $\mu \leq r \leq \bar{\mu}$, so there is a risk that the return from buying the stock is not as good as holding the bank account, and the investor would prefer not to invest in the stock. On the other hand, the best estimation of the drift $\bar{\mu}$ is still better than the interest rate $r$, so implementing a shortsale strategy may incur a potential loss for the investor. This refrains her from short selling the stock.

(v) Shortsale strategy When $\beta^{3} \leq 0$, the investor will hold short position in the stock as much as possible, which is $\max \left\{\beta_{3}, \underline{\pi}\right\}$ units of her wealth in this situation. Consequently, she keeps $\left(1-\max \left\{\beta_{3}, \underline{\pi}\right\}\right)$ units of her wealth in the bank account in order to earn the interest rate $r$.

We can further illustrate the above five optimal portfolio strategies via the following Fig. 1, where the horizontal axes represent the values of $\beta^{1}, \beta^{2}$ and $\beta^{3}$ from the top to the bottom, and the vertical axis represents the optimal portfolio.

In the existing literature, the worst-case parameters are usually bang-bang type, i.e., they take values at the boundaries of the uncertain parameter set. Next, we give an example where the worst-case drift and volatility are an interior point in the uncertain parameter set. In particular, the worst-case volatility may not be its upper bound anymore.

Assumption 4.3 Assume that $R=r, \bar{\pi}=+\infty, \underline{\pi}=-\infty$ and $\mathbb{B}=\{(\mu, \sigma): \mu=\mu+$ $\left.\alpha, \sigma=\underline{\sigma}^{2}+k \alpha^{q}, \alpha \in[0, \bar{\alpha}]\right\}$, where $\underline{\mu}, \underline{\sigma}, k, q, \bar{\alpha}$ are constants satisfying $\underline{\sigma} \geq 0, \bar{k}>$ $0,0<q<1, \bar{\alpha} \geq 0$.

The set $\mathbb{B}$ indicates that the ambiguities about drift and volatility are correlated. A higher return is associated with a larger risk. The limiting case $q=1$ means that the relationship between the ambiguity about drift and the ambiguity about the volatility square is linear, which is just Example 2.4 in [10]. The other spectrum $q=0$ means no ambiguity about volatility. Finally, $0<q<1$ means that the relationship between the ambiguity about drift and the ambiguity about the volatility square is sub-linear.

Theorem 4.4 Under Assumption 4.3, the worst-case parameters $\left(\mu^{*}, \sigma^{*}\right)$ and the optimal portfolio $\pi^{*}$ are given as follows: 


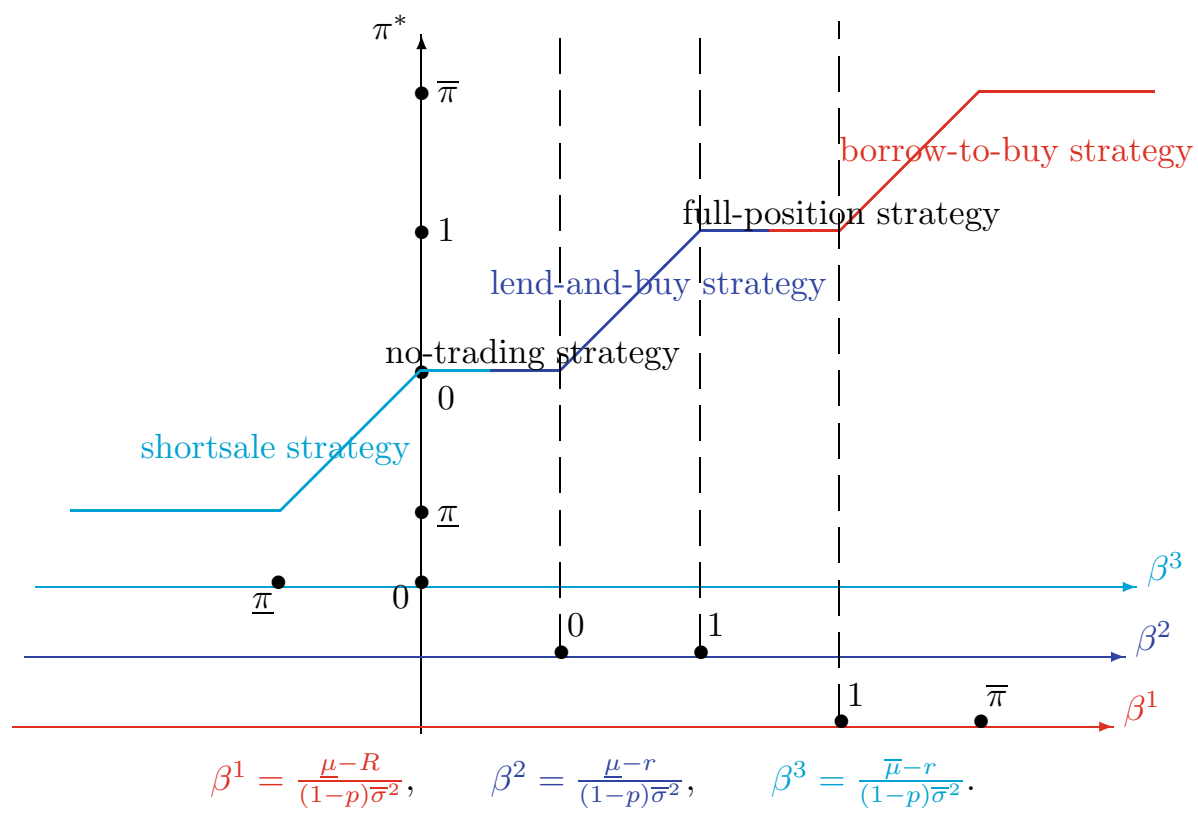

Fig. 1 The optimal portfolio strategies

(i) The worst-case parameters $\left(\mu^{*}, \sigma^{*}\right)=\left(\underline{\mu}+\alpha^{*}, \sqrt{\underline{\sigma}^{2}+k\left(\alpha^{*}\right)^{q}}\right)$, with

$$
\alpha^{*}= \begin{cases}r-\mu, & -\bar{\alpha}<\mu-r \leq 0 ; \\ \alpha_{0}, & 0<\underline{\mu}-r<\widehat{\alpha}:=\left[2 \underline{\sigma}^{2} \bar{\alpha}^{1-q}+k(2-q) \bar{\alpha}\right] /(k q) ; \\ \bar{\alpha}, & \text { otherwise, }\end{cases}
$$

where $\alpha_{0}$ is the solution of the following algebra equation (for the case $\mu-r>0$ ),

$$
h_{1}(\alpha):=2 \underline{\sigma}^{2}+k(2-q) \alpha^{q}-k q(\underline{\mu}-r) \alpha^{q-1}=0 ;
$$

(ii) The optimal portfolio $\pi^{*}$ is a constant process given by $\left(\mu^{*}-r\right) /\left((1-p)\left(\sigma^{*}\right)^{2}\right)$.

Proof First, we prove that the algebra equation (30) has a unique zero crossing point $\alpha_{0}$ for the case $\underline{\mu}-r>0$ and, moreover, $\alpha_{0} \in(0, \bar{\alpha})$ if $0<\underline{\mu}-r<\widehat{\alpha}$. Indeed, it is not difficult to check that for $\underline{\mu}-r>0$, we have

$$
\lim _{\alpha \rightarrow 0^{+}} h_{1}(\alpha)=-\infty, \quad \lim _{\alpha \rightarrow+\infty} h_{1}(\alpha)=+\infty,
$$

and

$$
h_{1}^{\prime}(\alpha)=k q(2-q) \alpha^{q-1}+k q(1-q)(\underline{\mu}-r) \alpha^{q-2}>0 .
$$

Hence, $h_{1}(\cdot)$ in the algebra equation (30) has a unique zero crossing point $\alpha_{0}$ for $\underline{\mu}-r>0$. Moreover, direct computations show that if $\underline{\mu}-r<\widehat{\alpha}$, then

$$
h_{1}(\bar{\alpha})=2 \underline{\sigma}^{2}+k(2-q) \bar{\alpha}^{q}-k q(\underline{\mu}-r) \bar{\alpha}^{q-1}>0,
$$

which means $\alpha_{0} \in(0, \bar{\alpha})$ if $0<\underline{\mu}-r<\widehat{\alpha}$. 
Secondly, we show that

$$
g\left(\pi^{*} ; \mu^{*},\left(\sigma^{*}\right)^{2}\right) \leq g\left(\pi^{*} ; x_{\mu}, x_{\sigma}\right), \quad\left(x_{\mu}, x_{\sigma}\right) \in \mathbb{B} .
$$

To this end, note that

$$
\begin{aligned}
g\left(\pi^{*} ; x_{\mu}, x_{\sigma}\right) & =\frac{p-1}{2}\left(\underline{\sigma}^{2}+k \alpha^{q}\right)\left(\pi^{*}\right)^{2}+(\underline{\mu}+\alpha-r) \pi^{*}+r \\
& =\frac{\left(\underline{\mu}+\alpha^{*}-r\right) h_{2}(\alpha)}{2(1-p)\left(\sigma^{*}\right)^{4}}+r,
\end{aligned}
$$

where

$$
h_{2}(\alpha):=-\left(\underline{\sigma}^{2}+k \alpha^{q}\right)\left(\underline{\mu}+\alpha^{*}-r\right)+2\left[\underline{\sigma}^{2}+k\left(\alpha^{*}\right)^{q}\right](\underline{\mu}+\alpha-r),
$$

and

$h_{2}^{\prime}(\alpha)=-k q \alpha^{q-1}\left(\underline{\mu}+\alpha^{*}-r\right)+2\left[\underline{\sigma}^{2}+k\left(\alpha^{*}\right)^{q}\right], \quad h_{2}^{\prime \prime}(\alpha)=k q(1-q) \alpha^{q-2}\left(\underline{\mu}+\alpha^{*}-r\right)$.

We divide the possible values of $\mu-r$ into four cases. Fix $\alpha \in[0, \bar{\alpha}]$ and $\left(x_{\mu}, x_{\sigma}\right) \in \mathbb{B}$. If $\underline{\mu}-r \leq-\bar{\alpha}$, then

$\underline{\mu}+\alpha^{*}-r \leq 0, \quad h_{2}^{\prime}(\alpha)>0, \quad h_{2}(\alpha) \leq h_{2}(\bar{\alpha})=h_{2}\left(\alpha^{*}\right), \quad g\left(\pi^{*} ; x_{\mu}, x_{\sigma}\right) \geq g\left(\pi^{*} ; \mu^{*},\left(\sigma^{*}\right)^{2}\right)$.

If $-\bar{\alpha}<\underline{\mu}-r \leq 0$, then

$\underline{\mu}+\alpha^{*}-r=\underline{\mu}+(r-\underline{\mu})-r=0, \quad \pi^{*}=0, \quad g\left(\pi^{*} ; x_{\mu}, x_{\sigma}\right)=r=g\left(\pi^{*} ; \mu^{*},\left(\sigma^{*}\right)^{2}\right)$.

If $0<\underline{\mu}-r<\widehat{\alpha}$, then

$$
\begin{aligned}
& \underline{\mu}+\alpha^{*}-r>0, \quad h_{2}^{\prime \prime}(\alpha)>0, \quad h_{2}^{\prime}\left(\alpha^{*}\right)=0, \quad h_{2}(\alpha) \geq h_{2}\left(\alpha^{*}\right), \\
& \quad g\left(\pi^{*} ; x_{\mu}, x_{\sigma}\right) \geq g\left(\pi^{*} ; \mu^{*},\left(\sigma^{*}\right)^{2}\right),
\end{aligned}
$$

where we have used the fact that $h_{1}\left(\alpha^{*}\right)=0$ implies that $h_{2}^{\prime}\left(\alpha^{*}\right)=0$. Finally, if $\underline{\mu}-r \geq \widehat{\alpha}$, then

$$
\begin{aligned}
& h_{2}^{\prime}(\bar{\alpha})=h_{1}(\bar{\alpha}) \leq 0, \quad h_{2}^{\prime \prime}(\alpha)>0, \quad h_{2}^{\prime}(\alpha) \leq 0, \quad h_{2}(\alpha) \geq h_{2}\left(\alpha^{*}\right), \\
& g\left(\pi^{*} ; x_{\mu}, x_{\sigma}\right) \geq g\left(\pi^{*} ; \mu^{*},\left(\sigma^{*}\right)^{2}\right) .
\end{aligned}
$$

Thirdly, we prove that

$$
g\left(\pi^{*} ; \mu^{*},\left(\sigma^{*}\right)^{2}\right) \geq g\left(x_{\pi} ; \mu^{*},\left(\sigma^{*}\right)^{2}\right), \quad x_{\pi} \in \mathbb{R} .
$$

To see this, we note that

$$
\begin{aligned}
g\left(x_{\pi} ; \mu^{*},\left(\sigma^{*}\right)^{2}\right) & =\frac{p-1}{2}\left(\sigma^{*}\right)^{2} x_{\pi}^{2}+\left(\mu^{*}-r\right) x_{\pi}+r \\
& =\frac{p-1}{2}\left(\sigma^{*}\right)^{2}\left[x_{\pi}-\frac{\mu^{*}-r}{(1-p)\left(\sigma^{*}\right)^{2}}\right]^{2}+\frac{\left(\mu^{*}-r\right)^{2}}{2(1-p)\left(\sigma^{*}\right)^{2}}+r .
\end{aligned}
$$

It is then clear that $g\left(x_{\pi} ; \mu^{*},\left(\sigma^{*}\right)^{2}\right)$ attains its maximum at the point $x_{\pi}=\pi^{*}$, so $\left(x_{\pi}^{*} ; \mu^{*},\left(\sigma^{*}\right)^{2}\right)$ is a saddle point of $g$, and the conclusion follows from Theorems 3.2 and 3.3. 
If $\mu-r \leq-\bar{\alpha}$, then the investor will short sell her stock, which is similar to Shortsale strategy in Theorem 4.2. Moreover, $\pi^{*}<0$ implies that the worst-case drift $\mu^{*}$ and volatility $\sigma^{*}$ attain their respective upper bounds $\mu+\bar{\alpha}$ and $\sqrt{\underline{\sigma}^{2}+k(\bar{\alpha})^{q}}$.

If $-\bar{\alpha}<\mu-r<0$, then the lower bound of the drift $\mu \leq r$ and the upper bound $\mu+\bar{\alpha}>r$. Similar to No-trading strategy in Theorem 4.2, the investor may suffer losses if she buys or short sells the stocks, so she will simply invest all her money in the bank account. Moreover, $\pi^{*}=0$ implies that the estimation of the drift and volatility is irrelevant in this situation and, without loss of generality, we let $\left(\mu^{*}, \sigma^{*}\right)=\left(r, \sqrt{\underline{\sigma}^{2}+k(r-\underline{\mu})^{q}}\right)$.

If $\mu-r>0$, then the investor will invest in the stock according to the optimal proportion $\pi^{*}=\left(\mu^{*}-r\right) /\left((1-p)\left(\sigma^{*}\right)^{2}\right)>0$. If there is no ambiguity about volatility, the worst-case drift is its lower bound $\mu$ and $\alpha^{*}=0$. Since the correlation between the uncertain drift and uncertain volatility is positive, the worst-case parameter $\alpha^{*}=\alpha_{0}$, which is an interior point of the interval $[0, \bar{\alpha}]$ if $0<\mu-r<\widehat{\alpha}$. In particular, the worst-case volatility may not be its upper bound anymore. This is in contrast to Theorem 4.2, where the worst-case parameters take values at the boundaries of the uncertain parameter set.

\subsection{The Optimal consumption under power utility}

In this section, we compute the explicit solutions to ODEs (15) and (19), which in turn allows us to construct the optimal consumption of the maxmin problem (5) [cf. (27) or (29)]. Note that if $\lambda=0$ in (4) and $\bar{c}=\underline{c}$, the consumption does not play a role and the optimal consumption strategy is simply $c_{t}^{*}=\bar{c}=\underline{c}$. Hence, we focus on the case $\lambda>0$ and $\bar{c}>\underline{c}$ in the rest of the paper. We first present the result for power utility.

Theorem 4.5 Let $T>0$ be a large enough number. For the power utility case, the optimal consumption $c_{t}^{*}=c^{*}(t), t \in[0, T]$, is a deterministic process, where $c^{*}(t)=\tilde{x}_{c, P}^{*}\left(q_{P}(t)\right)$ with $\tilde{x}_{c, P}^{*}(\cdot)$ given in (27) and $q_{P}(\cdot)$ given in Table 8. Moreover, the optimal consumption $c_{t}^{*}$ is summarized in Table 2. ${ }^{2}$

Herein, the constant $K$ in the table corresponds to the future investment contributing factor in (15), and has the explicit form

$$
K:=g\left(x_{\pi}^{*} ; x_{\mu}^{*}, x_{\sigma}^{*}\right)= \begin{cases}R+\bar{\pi}(\underline{\mu}-R)-\frac{1-p}{2} \bar{\sigma}^{2} \bar{\pi}^{2}, & \beta^{1} \geq \bar{\pi} ; \\ R+\frac{(\underline{\mu}-R)^{2}}{2(1-p) \bar{\sigma}^{2}}, & 1 \leq \beta^{1} \leq \bar{\pi} ; \\ \underline{\mu}-\frac{1-p}{2} \bar{\sigma}^{2}, & \beta^{1} \leq 1 \leq \beta^{2} ; \\ r+\frac{(\underline{\mu}-r)^{2}}{2(1-p) \bar{\sigma}^{2}}, & 0 \leq \beta^{2} \leq 1 ; \\ r, & \beta^{2} \leq 0 \leq \beta^{3} ; \\ r+\frac{(\bar{\mu}-r)^{2}}{2(1-p) \bar{\sigma}^{2}}, & \underline{\pi} \leq \beta^{3} \leq 0 ; \\ r+\underline{\pi}(\bar{\mu}-r)-\frac{1-p}{2} \bar{\sigma}^{2} \underline{\pi}^{2}, & \beta^{3} \leq \underline{\pi},\end{cases}
$$

and $\widehat{c}(t)=\widehat{c}_{P}\left(q_{P}(t)\right)[c f .(27)]$. The indicator function $I_{a}^{b}$ represents the time period $\left[T_{a}, T_{b}\right]$ with $T_{0}=0$ and $T_{4}=T$, where the explicit forms of different time periods are given in Appendix B.

${ }^{2}$ In the case of $\underline{c}=0$, the results are similar to those in Table 2 except that $\underline{c} I_{0}^{123}+\widehat{c}(t) I_{123}^{12}+\bar{c} I_{12}^{4}$ and $\underline{c} I_{0}^{23}+\widehat{c}(t) I_{23}^{4}$ are replaced by $\widehat{c}(t) I_{0}^{12}+\bar{c} I_{12}^{4}$ and $\widehat{c}(t)$, respectively. Note that when $\underline{c}=0$, since $\lambda>0$, the last two rows about the optimal consumption are then irrelevant. 
Table 2 The optimal consumption in the case of $\underline{c}>0$

\begin{tabular}{|c|c|c|c|c|c|}
\hline & $\begin{array}{c}\rho-p K \\
(-\infty,(1-p) \underline{c})\end{array}$ & $\{(1-p) \underline{c}\}$ & $((1-p) \underline{c},(1-p) \bar{c})$ & $\{(1-p) \bar{c}\}$ & $((1-p) \bar{c},+\infty)$ \\
\hline$\underline{c}<\bar{c}<\lambda^{1 /(1-p)}$ & $\underline{c} I_{0}^{123}+\widehat{c}(t) I_{123}^{12}+\bar{c} I_{12}^{4}$ & $\widehat{c}(t) I_{0}^{12}+\bar{c} I_{12}^{4}$ & $\widehat{c}(t) I_{0}^{12}+\bar{c} I_{12}^{4}$ & $\bar{c}$ & $\bar{c}$ \\
\hline$\underline{c}<\bar{c}=\lambda^{1 /(1-p)}$ & $\underline{c}_{0}^{23}+\widehat{c}(t) I_{23}^{4}$ & $\widehat{c}(t)$ & $\widehat{c}(t)$ & $\bar{c}$ & $\bar{c}$ \\
\hline$\underline{c}<\lambda^{1 /(1-p)}<\bar{c}$ & $\underline{c}_{0}^{23}+\widehat{c}(t) I_{23}^{4}$ & $\hat{c}(t)$ & $\widehat{c}(t)$ & $\hat{c}(t)$ & $\bar{c} I_{0}^{21}+\hat{c}(t) I_{21}^{4}$ \\
\hline$\lambda^{1 /(1-p)}=\underline{c}<\tau$ & $\underline{c}$ & $\underline{c}$ & $\hat{c}(t)$ & $\widehat{c}(t)$ & $\tau I_{0}^{21}+\hat{c}(t) I_{21}^{4}$ \\
\hline$\lambda^{1 /(1-p)}<\underline{c}<\bar{\tau}$ & $\underline{c}$ & $\underline{c}$ & $\widehat{c}(t) I_{0}^{32}+\underline{c} I_{32}^{4}$ & $\hat{c}(t) I_{0}^{32}+c_{32}^{4}$ & $\bar{c} I_{0}^{321}+\hat{c}(t) I_{321}^{32}+\underline{c}_{32}^{4}$ \\
\hline
\end{tabular}

Proof Due to its length, the proof is postponed to "Appendix B".

Table 2 lists all the possible consumption patterns under different parameters. For example, $\underline{c} I_{0}^{123}+\widehat{c}(t) I_{123}^{12}+\bar{c} I_{12}^{4}$ in the first row and the first column (left-top corner) is the optimal consumption when the market parameters satisfy $\underline{c}<\bar{c}<\lambda^{1 /(1-p)}$ and $\rho-p K \in(-\infty,(1-p) \underline{c})$. More specifically, in the time interval $\left[0, T_{123}\right]$, the investor will consume at the minimum rate $\underline{c}$. Then the investor will consume at the optimal rate $\widehat{c}(t)=\lambda^{1 /(1-p)} \exp \left(q_{P}(t) /(p-1)\right)$ in the time interval $\left[T_{123}, T_{12}\right]$, since in this case $\underline{c} \leq \widehat{c}(t) \leq \bar{c}$. Finally, in the remaining time interval $\left[T_{12}, T\right]$, the investor will consume at the maximum rate $\bar{c}$.

In contrast, in the right-bottom corner, we obtain a reversed consumption pattern when $\lambda^{1 /(1-p)}<\underline{c}<\bar{c}$ and $\rho-p K \in((1-p) \bar{c},+\infty)$. That is, the consumption will be decreasing from the maximum rate $\bar{c}$ in $\left[0, T_{321}\right]$, to $\widehat{c}(t)$ in $\left[T_{321}, T_{32}\right]$, and finally to the minimal rate $\underline{c}$ in $\left[T_{32}, T\right]$.

In the following, we give some intuitive explanations of different consumption patterns. From the expression of $f_{P}$ and $c^{*}(t)$, we know that the optimal consumption $c_{t}^{*}=c^{*}(t)$ achieves the maximum of the concave function $f_{P}\left(q_{P}(t), \cdot\right)$ in the interval $[\underline{c}, \bar{c}]$. Moreover, note that $\widehat{c}(t)=\lambda^{1 /(1-p)} \exp \left(q_{P}(t) /(p-1)\right)$ as in $(27)$ is the maximum point of $f_{P}\left(q_{P}(t), \cdot\right)$ on $\mathbb{R}_{+}$. Hence, $c_{t}^{*}=\widehat{c}(t)$ if $\underline{c}<\widehat{c}(t)<\bar{c}$. Otherwise, $c_{t}^{*}$ will be either $\underline{c}$ or $\bar{c}$.

From the proof of Proposition 4.6 below, we know $q_{P}(t)$ is monotone in time $t$, so is $\widehat{c}(t)$. As a result, whether $\widehat{c}(t)$ stays in $[\underline{c}, \bar{c}]$ or not only depends on its values at the two end points $\widehat{c}(T)$ and $\widehat{c}(0)$, and their relationship with $\underline{c}$ and $\bar{c}$.

In fact, it follows from $q_{P}(T)=0$ that $\widehat{c}(T)=1 / \lambda^{1-p}$. By the continuity of $\widehat{c}(t)$, when $t$ approaches maturity $T, c^{*}(t)$ will reach its upper bound $\bar{c}$ if $c<\bar{c}<\lambda^{1 /(1-p)} ; c^{*}(t)$ will be precisely $\widehat{c}(t)$ if $\underline{c}<\lambda^{1 /(1-p)}<\bar{c}$; $c^{*}(t)$ will reach its lower bound $\underline{c}$ if $\lambda^{1 /(1-p)}<\underline{c}<\bar{c}$. The above three situations thus determine the classification of the rows in Table 2 .

On the other hand, we have the following asymptotic results for $\lim _{T \rightarrow+\infty} \widehat{c}(0)$ in Table 3 (see also "Appendix B", in particular (49)-(52)). By the continuity of $\widehat{c}(t)$, when $T$ is large enough and $t$ is near initial time $0, c^{*}(t)=\underline{c}$ if $\rho-p K \in(-\infty,(1-p) \underline{c}) ; c^{*}(t)=\widehat{c}$ if $\rho-p K \in((1-p) \underline{c},(1-p) \bar{c})$; and $c^{*}(t)=\bar{c}$ if $\rho-p K \in((1-p) \bar{c},+\infty)$. Consequently, the above three situations divide the columns in Table 2 .

Next, we further show that the optimal consumption admits some time monotone properties. As opposed to the unconstrained consumption case, the consumption constraints may force the optimal consumption to be either nonincreasing or nondecreasing no matter the value of $(\rho-p K)$. 
Table 3 The limit of $\widehat{c}(0)$ when $T \rightarrow+\infty$

\begin{tabular}{llllll}
\hline$\rho-p K$ & $(-\infty,(1-p) \underline{c})$ & $\{(1-p) \underline{c}\}$ & $((1-p) \underline{c},(1-p) \bar{c})$ & $\{(1-p) \bar{c}\}$ & $((1-p) \bar{c},+\infty)$ \\
\hline $\lim _{T \rightarrow+\infty} \widehat{c}(0)$ & $<\underline{c}$ & $=\underline{c}$ & $\in(\underline{c}, \bar{c})$ & $=\bar{c}$ & $>\bar{c}$ \\
\hline
\end{tabular}

Table 4 The optimal consumption in time

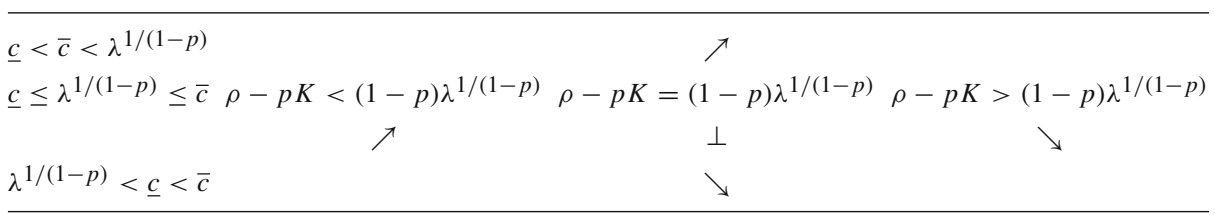

Proposition 4.6 The optimal consumption $c_{t}^{*}, t \in[0, T]$, has the following monotone properties in time $t$, as specified in Table 4. The symbols $\nearrow, \searrow$ and $\perp$ represent nondecreasing, nonincreasing and independent of time $t$, respectively.

Proof It follows from the expressions of $\widetilde{x}_{c, P}^{*}\left(x_{q}\right)$ and $\widehat{c}_{P}\left(x_{q}\right)$ in (27) that if $q_{P}(t)$ is nonincreasing, then $c^{*}(t)=\tilde{x}_{c, P}^{*}\left(q_{P}(t)\right)$ is nondecreasing; if $q_{P}(t)$ is nondecreasing, then $c^{*}(t)$ is nonincreasing. On the other hand, The expression (26) and ODE (15) lead to

$$
q_{P}^{\prime \prime}(t)=-p \bar{f}_{P}\left(q_{P}(t), c^{*}(t)\right) q_{P}^{\prime}(t),
$$

where

$\bar{f}_{P}\left(q_{P}(t), c^{*}(t)\right):= \begin{cases}-\frac{\lambda}{p} \bar{c}^{p} e^{-q_{P}(t)}<0, & \text { if } q_{P}(t)<(p-1) \ln \bar{c}+\ln \lambda ; \\ -\frac{\lambda^{1 /(1-p)}}{p} e^{q_{P}(t) /(p-1)}<0, & \text { if }(p-1) \ln \bar{c}+\ln \lambda \leq q_{P}(t) \leq(p-1) \ln \underline{c}+\ln \lambda ; \\ -\frac{\lambda}{p} \underline{c}^{p} e^{-q_{P}(t)}<0, & \text { if } q_{P}(t)>(p-1) \ln \underline{c}+\ln \lambda .\end{cases}$

We claim that the sign of $q_{P}^{\prime}(t)$ does not change for $t \in[0, T]$. Otherwise, suppose there exist $0 \leq t_{1}<t_{2} \leq T$ such that $q_{P}^{\prime}\left(t_{1}\right)>0$ and $q_{P}^{\prime}\left(t_{2}\right)<0$. By the continuity of $q_{P}^{\prime}(t)$, there exists $t \in\left(t_{1}, t_{2}\right)$ such that $q_{P}^{\prime}(t)=0$. Now let $t_{3}:=\inf \left\{t>t_{1}: q_{P}^{\prime}(t)=0\right\}$. It follows that $t_{3} \in\left(t_{1}, t_{2}\right), q_{P}^{\prime}\left(t_{3}\right)=0$, and $q_{P}^{\prime}(t)>0$ for $t \in\left[t_{1}, t_{3}\right)$. By the Mean Value Theorem, there exits $t_{4} \in\left(t_{1}, t_{3}\right)$ such that $q_{P}^{\prime \prime}\left(t_{4}\right)=\frac{q_{P}^{\prime}\left(t_{3}\right)-q_{P}^{\prime}\left(t_{1}\right)}{t_{3}-t_{1}}<0$. However, $q_{P}^{\prime \prime}(t)>0$ for $t \in\left[t_{1}, t_{3}\right)$ according to (32). This is a contradiction.

We have shown that $q_{P}(t)$ is either nonincreasing or nondecreasing for $t \in[0, T]$. Thus, it suffices to consider the sign of $q_{P}^{\prime}(T)$.

Let us first consider the case $\underline{c}<\bar{c}<\lambda^{1 /(1-p)}$. For this case, we have $(p-1) \ln \bar{c}+\ln \lambda>$ $0=q_{P}(T)$, and therefore, (26) implies that ODE (15) at $t=T$ reduces to

$$
q_{P}^{\prime}(T)=-\left(\lambda \bar{c}^{p}-p \bar{c}\right)-p K+\rho,
$$

where the constant $K$ is given in (31). However, Theorem 4.5 implies that $c^{*}(t) \equiv \bar{c}$ if $\rho-p K \geq(1-p) \bar{c}$ in this case, so we only need to consider the situation $\rho-p K<(1-p) \bar{c}$ for the monotone property of $c^{*}(t)$. Together with $\bar{c}<\lambda^{1 /(1-p)}$, we further obtain that

$$
q_{P}^{\prime}(T)<-\left(\bar{c}^{1-p} \bar{c}^{p}-p \bar{c}\right)+(1-p) \bar{c}=0 .
$$

In turn, $q_{P}^{\prime}(t) \leq 0$ for $t \in[0, T]$, which implies that $c^{*}(t)$ is nondecreasing for $t \in[0, T]$.

The other two cases $\underline{c} \leq \lambda^{1 /(1-p)} \leq \bar{c}$ and $\lambda^{1 /(1-p)}<\underline{c}<\bar{c}$ can be treated in a similar way, so their proofs are omitted. 
Table 5 The optimal consumption in the case of $\underline{c} \geq 0$

\begin{tabular}{lccccc}
\hline & $0<\rho<\underline{c}$ & $\rho=\underline{c}$ & $\underline{c}<\rho<\bar{c}$ & $\rho=\bar{c}$ & $\rho>\bar{c}$ \\
\hline $0<\lambda \leq \underline{c}$ & $\underline{c}$ & $\underline{c}$ & $\widehat{c}(t) I_{0}^{2}+\underline{c} I_{2}^{3}$ & $\widehat{c}(t) I_{0}^{2}+\underline{c} I_{2}^{3}$ & $\bar{c} I_{0}^{1}+\widehat{c}(t) I_{1}^{2}+\underline{c} I_{2}^{3}$ \\
\hline$\underline{c}<\lambda<\bar{c}$ & $\underline{c} I_{0}^{2}+\widehat{c}(t) I_{2}^{3}$ & $\hat{c}(t)$ & $\hat{c}(t)$ & $\hat{c}(t)$ & $\bar{c} I_{0}^{1}+\widehat{c}(t) I_{1}^{3}$ \\
\hline$\lambda \geq \bar{c}$ & $\underline{c} I_{0}^{2}+\widehat{c}(t) I_{2}^{1}+\bar{c} I_{1}^{3}$ & $\widehat{c}(t) I_{0}^{1}+\bar{c} I_{1}^{3}$ & $\widehat{c}(t) I_{0}^{1}+\bar{c} I_{1}^{3}$ & $\bar{c}$ & $\bar{c}$ \\
\hline
\end{tabular}

\subsection{The optimal consumption under logarithm utility}

Theorem 4.7 Assume that $T$ is a large enough number. For the logarithm utility case, the optimal consumption $c_{t}^{*}=\tilde{x}_{c, L}^{*}\left(q_{L}(t)\right), t \in[0, T]$, is a deterministic process, with $\tilde{x}_{c, L}^{*}(\cdot)$ and $q_{L}(\cdot)$ given respectively in (29) and (34). Moreover, the optimal consumption $c_{t}^{*}$ is summarized in Table $5 .^{3}$

Herein, $I_{b}^{a}$ represents the indicator function of the time interval $\left[T_{a}, T_{b}\right]$, and

$$
\begin{array}{ll}
\widehat{c}(t)=\lambda e^{-q_{L}(t)}, \quad T_{0}=0, & T_{1}=T+\frac{1}{\rho} \ln \frac{\lambda(\rho-\bar{c})}{\bar{c}(\rho-\lambda)}, \\
T_{2}=T+\frac{1}{\rho} \ln \frac{\lambda(\rho-\underline{c})}{\underline{c}(\rho-\lambda)}, & T_{4}=T .
\end{array}
$$

The function $q_{L}$ takes the form

$$
q_{L}(t)=\ln \left[\frac{\lambda}{\rho}+\left(1-\frac{\lambda}{\rho}\right) e^{-\rho(T-t)}\right] .
$$

Moreover, the optimal consumption $c_{t}^{*}$ is nonincreasing with respect to $t$ for $\rho \geq \lambda$, and nondecreasing with respect to $t$ for $\rho \leq \lambda$.

Proof First, it is clear that the solution of ODE (19) takes the form (34). From (29), we know that $\tilde{x}_{c, L}^{*}\left(x_{q}\right)$ is nonincreasing with respect to $x_{q}$. Moreover, the expression (34) implies that $q_{L}(\cdot)$ is nondecreasing with respect to $t$ when $\rho \geq \lambda$, and nonincreasing with respect to $t$ when $\rho \leq \lambda$. Then, the monotonicity of $c_{t}^{*}=\tilde{x}_{c, L}^{*}\left(q_{L}(t)\right)$ follows immediately.

Next, we note that

$$
e^{q_{L}(T)}=1\left\{\begin{array}{ll}
\geq \frac{\lambda}{c}, & 0<\lambda \leq \underline{c} ; \\
\in\left(\frac{\lambda}{\bar{c}}, \frac{\lambda}{c}\right), & \underline{c}<\lambda<\bar{c} ; \\
\leq \frac{\lambda}{\bar{c}}, & \lambda \geq \bar{c},
\end{array} \quad \lim _{\rightarrow \rightarrow+\infty} e^{q_{L}(0)}=\frac{\lambda}{\rho} \begin{cases}\geq \frac{\lambda}{c}, & 0<\rho \leq c \\
\in\left(\frac{\lambda}{\bar{c}}, \frac{\lambda}{c}\right), & \underline{c}<\rho<\bar{c} ; \\
\leq \frac{\lambda}{\bar{c}}, & \rho \geq \bar{c} .\end{cases}\right.
$$

In the following, we only prove the case $0<\lambda \leq \underline{c}$ and $\rho>\bar{c}$. Other cases follow along similar arguments. It follows from (35) that

$$
\begin{aligned}
& \widehat{c}(T)=\lambda e^{-q_{L}(T)} \leq \underline{c}<\bar{c}<\lambda e^{-q_{L}(0)}=\widehat{c}(0), \quad c_{T}^{*}=\tilde{x}_{c, L}^{*}\left(q_{L}(T)\right)=\underline{c}, \\
& c_{0}^{*}=\tilde{x}_{c, L}^{*}\left(q_{L}(0)\right)=\bar{c}
\end{aligned}
$$

provided $T$ is large enough. Moreover, since $q_{L}(\cdot)$ is continuous and strictly increasing with respect to $t$, there exists unique $\left(T_{1}, T_{2}\right)$ such that

\footnotetext{
3 Note that when $\underline{c}=0$, since $\lambda>0$ and $\rho \geq 0$, the first row and the first column about the optimal consumption are then irrelevant.
} 
Table 6 The comparative statistics

\begin{tabular}{cccccccccc}
\hline & $R$ & $\underline{\pi}$ & $\bar{\pi}$ & $\underline{c}$ & $\bar{c}$ & $\underline{\mu}$ & $\bar{\mu}$ & $\underline{\sigma}$ & $\bar{\sigma}$ \\
\hline$\mu_{s}^{*}$ & $\perp$ & $\perp$ & $\perp$ & $\perp$ & $\perp$ & $\nearrow$ & $\nearrow$ & $\perp$ & $\perp$ \\
$\sigma_{s}^{*}$ & $\perp$ & $\perp$ & $\perp$ & $\perp$ & $\perp$ & $\perp$ & $\perp$ & $\perp$ & $\nearrow$ \\
$\pi_{s}^{*}$ & $\searrow$ & $\nearrow$ & $\nearrow$ & $\perp$ & $\perp$ & $\nearrow$ & $\nearrow$ & $\perp$ & $\searrow$ \\
$c_{s}^{*}$ & $\nearrow$ & $\nearrow$ & $\searrow$ & $\nearrow$ & $\mathrm{NM}$ & $\searrow$ & $\nearrow$ & $\perp$ & $\nearrow$ \\
\hline
\end{tabular}

$$
\begin{aligned}
& q_{L}(t) \geq \ln \frac{\lambda}{\frac{c}{c}}, \quad t \in\left[T_{2}, T\right] ; \quad \ln \frac{\lambda}{\bar{c}}<q_{L}(t)<\ln \frac{\lambda}{\underline{c}}, \quad t \in\left(T_{1}, T_{2}\right) ; \\
& q_{L}(t) \leq \ln \frac{\lambda}{\bar{c}}, \quad t \in\left[0, T_{1}\right],
\end{aligned}
$$

and $T_{1}, T_{2}$ take the form in (33). Together with (29), we deduce that $c_{t}^{*}=\tilde{x}_{c, L}^{*}\left(q_{L}(t)\right)=$ $\bar{c} I_{0}^{1}+\widehat{c}(t) I_{1}^{2}+\underline{c} I_{2}^{3}$.

\section{The impacts of model uncertainty, portfolio-consumption constraints and borrowing costs}

In this section, we investigate the impacts of model uncertainty, portfolio-consumption constraints and borrowing costs on the worst-case parameters $\left(\mu^{*}, \sigma^{*}\right)$ and the optimal portfolio-consumption strategies $\left(\pi^{*}, c^{*}\right)$.

Proposition 5.1 Suppose that Assumption 4.1 holds. Then, for the power utility case, the worst-case parameters and the optimal portfolio-consumption strategies admit the following monotone properties in terms of the borrowing rate $R$, the constraint set $[\underline{\pi}, \bar{\pi}] \times[\underline{c}, \bar{c}]$, and the uncertain parameter set $[\mu, \bar{\mu}] \times\left[\underline{\sigma}^{2}, \bar{\sigma}^{2}\right]$, as specified in Table 6 . The symbols $\searrow$, $\nearrow, \perp$ and NM represent nonincreasing, nondecreasing, independent and non-monotone of the corresponding variable. For example, the bottom row and the first column (left-bottom corner) means $c_{s}^{*}$ is nondecreasing in the borrowing rate $R$.

Before proceeding to the proof, we provide some intuitive explanations for the above results. The impacts of different parameters on the worst-case parameters $\left(\mu_{s}^{*}, \sigma_{s}^{*}\right)$ and the optimal portfolio $\pi_{s}^{*}$ are obvious from the results in Theorem 4.2. So we only discuss about their impacts on the optimal consumption $c_{s}^{*}$.

By the expression (27) and $c_{s}^{*}=\tilde{x}_{c, P}^{*}\left(q_{P}(s)\right)$, the parameters $(R, \underline{\pi}, \bar{\pi}, \underline{\mu}, \bar{\mu}, \underline{\sigma}, \bar{\sigma})$ will effect the optimal consumption through the channel of the opportunity process $e^{q_{P}(s)}$, which is the investor's extra utilities obtained by optimizing over all the admissible portfolioconsumption strategies (least affected by model uncertainty) in the remaining horizon $[s, T]$. A closer look at the ODE (15) for $q_{P}(s)$ tells us that those parameters will only enter into the future investment contributing factor $g\left(x_{\pi}^{*} ; x_{\mu}^{*}, x_{\sigma}^{*}\right)$ in (23). Increasing the borrowing $\operatorname{cost} R$ will make the future investment contributing factor $g\left(x_{\pi}^{*} ; x_{\mu}^{*}, x_{\sigma}^{*}\right)$ smaller, so the opportunity process will also become smaller, i.e. the investor will obtain less utilities in the remaining horizon. In turn, her current optimal consumption will go up. Similarly, enlarging the uncertainty parameters interval $[\mu, \bar{\mu}] \times\left[\underline{\sigma}^{2}, \bar{\sigma}^{2}\right]$ or shrinking the portfolio constraint interval $[\underline{\pi}, \bar{\pi}]$ will also make the future investment contributing factor $g\left(x_{\pi}^{*} ; x_{\mu}^{*}, x_{\sigma}^{*}\right)$ smaller, and therefore, the current optimal consumption will arise.

The more striking result is probably the impact of the consumption constraint interval $[\underline{c}, \bar{c}]$ on the optimal consumption $c_{s}^{*}$. Note that the constraint interval will only effect the 
consumption contributing factor $f_{P}\left(q_{P}(s), c^{*}(s)\right)$ in (22), with $c^{*}(s)=\tilde{x}_{c, P}^{*}\left(q_{P}(s)\right)$. The smaller interval will lead to a smaller consumption contributing factor $f_{P}\left(q_{P}(s), c^{*}(s)\right)$ as indicated in (24). In turn, the investor will obtain less utilities in the remaining horizon $[s, T]$. This might suggest the current optimal consumption would increase. However, it is not always the case, as there is less chance for the unconstrained optimal consumption $\widehat{c}(s)$ to stay in the shrinking interval $[\underline{c}, \bar{c}]$. If $\widehat{c}(s)$ reaches the lower bound $\underline{c}$, then the optimal consumption will further arise as $\underline{c}$ increases. On the other hand, if $\widehat{c}(s)$ reaches the upper bound $\bar{c}$, then the optimal consumption will go down for $\bar{c}$ becomes smaller, thus offsets the previous increasing impact on the optimal consumption when $\bar{c}$ is decreasing. This means the optimal consumption is non-monotone in its upper bound $\bar{c}$.

Proof (i) The monotone property of $\mu_{s}^{*}$ According to Theorem 4.2, the worst-case drift can be rewritten as

$$
\begin{aligned}
\mu_{s}^{*} & =\underline{\mu} 1_{\{\bar{\mu} \geq r\}}+\bar{\mu} 1_{\{\bar{\mu}<r\}} \\
& =\underline{\mu} 1_{\{\underline{\mu}>r\}}+\bar{\mu} 1_{\{\underline{\mu} \leq r\}}
\end{aligned}
$$

for $s \in[0, T]$. The first line implies that $\mu_{s}^{*}$ is nondecreasing in $\mu$, and the second line implies it is also nondecreasing in $\bar{\mu}$, and is irrelevant to the other parameters $(R, \underline{\pi}, \bar{\pi}, \underline{c}, \bar{c}, \underline{\sigma}, \bar{\sigma})$.

(ii) The monotone property of $\sigma_{s}^{*}$ The conclusion simply follows from the expression of the worst-case volatility $\sigma_{s}^{*}=\bar{\sigma}$ for $s \in[0, T]$ in Theorem 4.2.

(iii) The monotone property of $\pi_{s}^{*}$ First, the expressions of $\beta^{1}, \beta^{2}, \beta^{3}$ in Theorem 4.2 imply that they are all nondecreasing in $\mu, \bar{\mu}$, nonincreasing in $R, \bar{\sigma}$ and independent of $\underline{\sigma}, \underline{c}, \bar{c}$, so is the optimal portfolio $\pi_{s}^{*}$, as $\pi_{s}^{*}$ is nondecreasing with respect to $\beta^{1}, \beta^{2}, \beta^{3}$ (cf. Fig. 1).

From Table 1 in Theorem 4.2, we further obtain

$$
\begin{aligned}
\pi_{s}^{*} & =\min \left\{\beta^{1}, \bar{\pi}\right\} 1_{\left\{\beta^{1} \geq 1\right\}}+C_{1} 1_{\left\{\beta^{1}<1\right\}} \\
& =\max \left\{\beta^{3}, \underline{\pi}\right\} 1_{\left\{\beta^{3} \leq 0\right\}}+C_{2} 1_{\left\{\beta^{3}>0\right\}}
\end{aligned}
$$

for some constants $C_{1}$ independent of $\bar{\pi}$, and $C_{2}$ independent of $\pi$. Hence, $\pi_{s}^{*}$ is nondecreasing in both $\underline{\pi}$ and $\bar{\pi}$.

(iv) The monotone property of $c_{s}^{*}$ We first study the impacts of different parameters on the solution $q_{P}(t)$ of $\operatorname{ODE}(15)$. Note that $(R, \underline{\pi}, \bar{\pi}, \underline{\mu}, \bar{\mu}, \underline{\sigma}, \bar{\sigma})$ will effect $q_{P}(t)$ only through $g\left(x_{\pi}^{*} ; x_{\mu}^{*}, x_{\sigma}^{*}\right)=K$, where $K$ is given in (31).

It is obvious from the expression (31) that $K$ is nonincreasing in $R$. Moreover, since $K$ is the maximum value of $g\left(x_{\pi} ; x_{\mu}^{*}, x_{\sigma}^{*}\right)$ over $x_{\pi} \in[\underline{\pi}, \bar{\pi}], K$ is nonincreasing in $\underline{\pi}$ and nondecreasing in $\bar{\pi}$. On the other hand, $K$ is also the minimum value of $g\left(x_{\pi}^{*} ; x_{\mu}, x_{\sigma}\right)$ over $\left(x_{\mu}, x_{\sigma}\right) \in[\underline{\mu}, \bar{\mu}] \times\left[\underline{\sigma}^{2}, \bar{\sigma}^{2}\right]$. Therefore, $K$ is nondecreasing in $\underline{\mu}, \underline{\sigma}$ and nonincreasing in $\bar{\mu}, \bar{\sigma}$. However, the expression of $K$ further implies that $K$ is independent of $\underline{\sigma}$.

It then follows from the comparison theorem for ODE (15) that its solution $q_{P}(s)$ is nonincreasing in $R, \underline{\pi}, \bar{\mu}, \bar{\sigma}$, nondecreasing in $\bar{\pi}$ and $\mu$, and independent of $\underline{\sigma}$. The conclusion about the optimal consumption $c_{s}^{*}$ then follows from Theorem 3.2 together with the expression (27).

In terms of the impacts of $\underline{c}$ and $\bar{c}$ on $c_{s}^{*}$, since $f_{P}\left(q_{P}(s), c^{*}(s)\right)$, with $c^{*}(s)=\tilde{x}_{c, P}^{*}\left(q_{P}(s)\right)$, is the maximum value of $f_{P}\left(q_{P}(s), x_{c}\right)$ over $x_{c} \in[\underline{c}, \bar{c}]$, it is nonincreasing in $\underline{c}$ and nondecreasing in $\bar{c}$. Following the comparison theorem for ODE (15) and the expression (27) once again, we conclude $\widehat{c}(s)$ is nondecreasing in $\underline{c}$ and nonincreasing in $\bar{c}$.

In turn, the expression (27) implies that the optimal consumption $c_{s}^{*}$ is also nondecreasing in $\underline{c}$, but neither increasing nor decreasing in $\bar{c}$, for the second and last terms in $c^{*}(s)$ offset 
Table 7 The comparative statistics for $\log$

\begin{tabular}{cccccccccc}
\hline & $R$ & $\underline{\pi}$ & $\bar{\pi}$ & $\underline{c}$ & $\bar{c}$ & $\underline{\mu}$ & $\bar{\mu}$ & $\underline{\sigma}$ & $\bar{\sigma}$ \\
\hline$\mu^{*}$ & $\perp$ & $\perp$ & $\perp$ & $\perp$ & $\perp$ & $\nearrow$ & $\nearrow$ & $\perp$ & $\perp$ \\
$\sigma^{*}$ & $\perp$ & $\perp$ & $\perp$ & $\perp$ & $\perp$ & $\perp$ & $\perp$ & $\perp$ & $\nearrow$ \\
$\pi^{*}$ & $\searrow$ & $\nearrow$ & $\nearrow$ & $\perp$ & $\perp$ & $\nearrow$ & $\nearrow$ & $\perp$ & $\searrow$ \\
$c^{*}$ & $\perp$ & $\perp$ & $\perp$ & $\nearrow$ & $\nearrow$ & $\perp$ & $\perp$ & $\perp$ & $\perp$ \\
\hline
\end{tabular}

the effects of each other. Indeed, we show the non-monotonicity in the case of $0 \leq \underline{c}<\bar{c}_{2}<$ $\bar{c}_{1}<\lambda^{1 /(1-p)}$ and $\rho-p K \in\left((1-p) \underline{c},(1-p) \bar{c}_{2}\right)$. According to Theorem 4.5, both $c_{1}^{*}(t)$ and $c_{2}^{*}(t)$ take the form of $\widehat{c}(t) I_{0}^{12}+\bar{c} I_{12}^{4}$. When $t$ is close to $T$, then $c_{1}^{*}(t)=\bar{c}_{1}>\bar{c}_{2}=c_{2}^{*}(t)$. On the other hand, when $T$ is large enough and $t$ is close to zero, we have

$$
c_{1}^{*}(t)=\widehat{c}_{1}(t)=\exp \left\{\frac{q_{P, 1}(t)}{p-1}\right\}<\exp \left\{\frac{q_{P, 2}(t)}{p-1}\right\}=\widehat{c}_{2}(t)=c_{2}^{*}(t),
$$

where the strict inequality can be derived from the comparison theorem for ODE.

Finally, we present the result for the logarithm utility case. Its proof is omitted as it is similar to the proof for the power utility case.

Proposition 5.2 Suppose that Assumption 4.1 holds. Then, for the logarithm utility case, the worst-case parameters and the optimal portfolio-consumption strategies have the following monotone properties in terms of the borrowing rate $R$, the constraint set $[\underline{\pi}, \bar{\pi}] \times[\underline{c}, \bar{c}]$, and the uncertain parameter set $[\mu, \bar{\mu}] \times\left[\underline{\sigma}^{2}, \bar{\sigma}^{2}\right]$, as showed in Table 7. The symbols $\searrow, \nearrow, \perp$ represent nonincreasing, nondecreasing and independent of the corresponding variable.

Open Access This article is distributed under the terms of the Creative Commons Attribution 4.0 International License (http://creativecommons.org/licenses/by/4.0/), which permits unrestricted use, distribution, and reproduction in any medium, provided you give appropriate credit to the original author(s) and the source, provide a link to the Creative Commons license, and indicate if changes were made.

\section{A Appendix: Proof of Theorem 4.2}

Proof of Theorem 4.2 According to Theorem 3.2, if $\left\{x_{\pi}^{*} ; x_{\mu}^{*}, x_{\sigma}^{*}\right\}$ is a saddle point of the function $g(\cdot ; \cdot, \cdot)$, then $x_{\pi}^{*}$ is the optimal portfolio, and $\left(x_{\mu}^{*}, \sqrt{x_{\sigma}^{*}}\right)$ are the worst-case parameters. Thus, it is sufficient to show that $\left\{\pi^{*} ; \mu^{*}, x_{\sigma}^{*}\right\}$ given in Theorem 4.2 is indeed a saddle point of the function $g(\cdot ; \cdot, \cdot)$.

First, for fixed $x_{\pi} \in[\underline{\pi}, \bar{\pi}]$, it is obvious to check that with

$$
g\left(x_{\pi} ; x_{\mu}, x_{\sigma}\right)=\frac{p-1}{2} x_{\sigma} x_{\pi}^{2}+\left(x_{\mu} x_{\pi}+r\left(1-x_{\pi}\right)-(R-r)\left(1-x_{\pi}\right)^{-}\right),
$$

we have

$$
\min _{\left(x_{\mu}, x_{\sigma}\right) \in[\underline{\mu}, \bar{\mu}] \times\left[\underline{\sigma}^{2}, \bar{\sigma}^{2}\right]} g\left(x_{\pi} ; x_{\mu}, x_{\sigma}\right)= \begin{cases}g\left(x_{\pi} ; \underline{\mu}, \bar{\sigma}^{2}\right), & \text { if } x_{\pi}>0 ; \\ \left.g\left(x_{\pi} ; \underline{\mu}, \bar{\mu}\right], \bar{\sigma}^{2}\right), & \text { if } x_{\pi}=0 ; \\ g\left(x_{\pi} ; \bar{\mu}, \bar{\sigma}^{2}\right), & \text { if } x_{\pi}<0,\end{cases}
$$

where $[\underline{\mu}, \bar{\mu}]$ means that $x_{\mu}^{*}$ may take any value in that interval. 
The above minimum function can be further written in a compact form by defining

$$
\begin{aligned}
g_{1}\left(x_{\pi}\right):= & g\left(x_{\pi} ; x_{\mu}^{*}, x_{\sigma}^{*}\right) \\
= & \frac{p-1}{2} \bar{\sigma}^{2} x_{\pi}^{2}+\left(\underline{\mu} I_{\left\{x_{\pi}>0\right\}}+\bar{\mu} I_{\left\{x_{\pi}<0\right\}}-r I_{\left\{x_{\pi}<1\right\}}-R I_{\left\{x_{\pi} \geq 1\right\}}\right) x_{\pi} \\
& +\left(r I_{\left\{x_{\pi}<1\right\}}+R I_{\left\{x_{\pi} \geq 1\right\}}\right) .
\end{aligned}
$$

In the following, we study the maximum value of $g_{1}\left(x_{\pi}\right)$ in three different cases $x_{\pi} \geq 1$, $0 \leq x_{\pi} \leq 1$ and $x_{\pi} \leq 0$, then together with the constraint $\underline{\pi} \leq x_{\pi} \leq \bar{\pi}$, we will obtain the maximizer $x_{\pi}^{*}$ and the associated maximum value $g_{1}\left(x_{\pi}^{*}\right)$.

Case (1) $x_{\pi} \geq 1$.

$$
g_{1}\left(x_{\pi}\right)=\frac{p-1}{2} \bar{\sigma}^{2}\left[x_{\pi}+\frac{\underline{\mu}-R}{(p-1) \bar{\sigma}^{2}}\right]^{2}+R-\frac{(\underline{\mu}-R)^{2}}{2(p-1) \bar{\sigma}^{2}} .
$$

If $\beta_{1}=(\mu-R) /\left((1-p) \bar{\sigma}^{2}\right) \geq \bar{\pi}$, then

$$
\max _{1 \leq x_{\pi} \leq \bar{\pi}} g_{1}\left(x_{\pi}\right)=g_{1}(\bar{\pi}) \geq g_{1}(1) .
$$

If $1<\beta_{1}<\bar{\pi}$, then

$$
\max _{1 \leq x_{\pi} \leq \bar{\pi}} g_{1}\left(x_{\pi}\right)=g_{1}\left(\beta_{1}\right)>g_{1}(1) .
$$

If $\beta_{1} \leq 1$, then

$$
\max _{1 \leq x_{\pi} \leq \bar{\pi}} g_{1}\left(x_{\pi}\right)=g_{1}(1) .
$$

Case (2) $0 \leq x_{\pi} \leq 1$.

$$
g_{1}\left(x_{\pi}\right)=\frac{p-1}{2} \bar{\sigma}^{2}\left[x_{\pi}+\frac{\underline{\mu}-r}{(p-1) \bar{\sigma}^{2}}\right]^{2}+r-\frac{(\underline{\mu}-r)^{2}}{2(p-1) \bar{\sigma}^{2}} .
$$

If $\beta_{2}=(\underline{\mu}-r) /\left((1-p) \bar{\sigma}^{2}\right) \geq 1$, then

$$
\max _{0 \leq x_{\pi} \leq 1} g_{1}\left(x_{\pi}\right)=g_{1}(1)>g_{1}(0) .
$$

If $0<\beta_{2}<1$, then

$$
\max _{0 \leq x_{\pi} \leq 1} g_{1}\left(x_{\pi}\right)=g_{1}\left(\beta_{2}\right)>\max \left\{g_{1}(1), g_{1}(0)\right\} .
$$

If $\beta_{2} \leq 0$, then

$$
\max _{0 \leq x_{\pi} \leq 1} g_{1}\left(x_{\pi}\right)=g_{1}(0)>g_{1}(1) .
$$

Case (3) $x_{\pi} \leq 0$.

$$
g_{1}\left(x_{\pi}\right)=\frac{p-1}{2} \bar{\sigma}^{2}\left[x_{\pi}+\frac{\bar{\mu}-r}{(p-1) \bar{\sigma}^{2}}\right]^{2}+r-\frac{(\bar{\mu}-r)^{2}}{2(p-1) \bar{\sigma}^{2}} .
$$

If $\beta_{3}=(\bar{\mu}-r) /\left((1-p) \bar{\sigma}^{2}\right) \geq 0$, then

$$
\max _{\underline{\pi} \leq x_{\pi} \leq 0} g_{1}\left(x_{\pi}\right)=g_{1}(0) .
$$


Table 8 The explicit solution $q_{P}(\cdot)$ to ODE (15) in the case of $\underline{c}>0$

\begin{tabular}{lccccc}
\hline & $\rho-p K$ & & & \\
& $(-\infty,(1-p) \underline{c})$ & $\{(1-p) \underline{c}\}$ & $((1-p) \underline{c},(1-p) \bar{c})$ & $\{(1-p) \bar{c}\}$ & $((1-p) \bar{c},+\infty)$ \\
\hline$\underline{c}<\bar{c}<\lambda^{1 /(1-p)}$ & $q_{123}(t)$ & $q_{12}(t)$ & $q_{12}(t)$ & $q_{1}(t)$ & $q_{1}(t)$ \\
$\underline{c}<\bar{c}=\lambda^{1 /(1-p)}$ & $q_{23}(t)$ & $q_{2}(t)$ & $q_{2}(t)$ & $q_{1}(t)$ & $q_{1}(t)$ \\
$\underline{c}<\lambda^{1 /(1-p)}<\bar{c}$ & $q_{23}(t)$ & $q_{2}(t)$ & $q_{2}(t)$ & $q_{2}(t)$ & $q_{21}(t)$ \\
$\lambda^{1 /(1-p)}=\underline{c}<\bar{c}$ & $q_{3}(t)$ & $q_{3}(t)$ & $q_{2}(t)$ & $q_{2}(t)$ & $q_{21}(t)$ \\
$\lambda^{1 /(1-p)}<\underline{c}<\bar{c}$ & $q_{3}(t)$ & $q_{3}(t)$ & $q_{32}(t)$ & $q_{32}(t)$ & $q_{321}(t)$ \\
\hline
\end{tabular}

If $\underline{\pi}<\beta_{3}<0$, then

$$
\max _{\underline{\pi} \leq x_{\pi} \leq 0} g_{1}\left(x_{\pi}\right)=g_{1}\left(\beta_{3}\right)>g_{1}(0)
$$

If $\beta_{3} \leq \underline{\pi}$, then

$$
\max _{\underline{\pi} \leq x_{\pi} \leq 0} g_{1}\left(x_{\pi}\right)=g_{1}(\underline{\pi}) \geq g_{1}(0) .
$$

Comparing the maximum values in the above three cases, and noting that the fact $\beta_{1} \leq$ $\beta_{2} \leq \beta_{3}$, we see that $\max _{\underline{\pi} \leq x_{\pi} \leq \bar{\pi}} g_{1}\left(x_{\pi}\right)=g_{1}\left(x_{\pi}^{*}\right)=K$, where $K$ is defined in (31), and the optimal $x_{\pi}^{*}$ is defined in Table 1 . Thus, we have proved

$$
g\left(x_{\pi}^{*} ; x_{\mu}^{*}, x_{\sigma}^{*}\right) \geq g\left(x_{\pi} ; x_{\mu}^{*}, x_{\sigma}^{*}\right), \quad \forall x_{\pi} \in[\underline{\pi}, \bar{\pi}] .
$$

On the other hand, with $x_{\pi}^{*}$ as in Table 1, it follows from (36) that

$$
g\left(x_{\pi}^{*} ; x_{\mu}^{*}, x_{\sigma}^{*}\right) \leq g\left(x_{\pi}^{*} ; x_{\mu}, x_{\sigma}\right), \forall\left(x_{\mu}, x_{\sigma}\right) \in[\underline{\mu}, \bar{\mu}] \times\left[\underline{\sigma}^{2}, \bar{\sigma}^{2}\right] .
$$

Hence, $\left\{x_{\pi}^{*} ; x_{\mu}^{*}, x_{\sigma}^{*}\right\}$ is a saddle point of the function $g(\cdot ; \cdot, \cdot)$.

\section{B Appendix: Proof of Theorem 4.5}

First, we give the explicit solution to ODE (15) in Table 8 when $\underline{c}>0 .{ }^{4}$

The solutions $q_{123}, q_{12}, q_{1}, q_{23}, q_{2}, q_{21}, q_{3}, q_{32}, q_{321}$ have the explicit forms

$$
\begin{aligned}
q_{123}(t)= & q^{1}\left(t ; 1 / \lambda, T_{12}, T\right) I_{\left[T_{12}, T\right]}+q^{2}\left(t ; \bar{c}^{p-1}, T_{123}, T_{12}\right) I_{\left[T_{123}, T_{12}\right]} \\
& +q^{3}\left(t ; \underline{c}^{p-1}, 0, T_{123}\right) I_{\left[0, T_{123}\right]} ; \\
q_{12}(t)= & q^{1}\left(t ; 1 / \lambda, T_{12}, T\right) I_{\left[T_{12}, T\right]}+q^{2}\left(t ; \bar{c}^{p-1}, 0, T_{12}\right) I_{\left[0, T_{12}\right]} ; \\
q_{1}(t)= & q^{1}(t ; 1 / \lambda, 0, T) ; \quad q_{2}(t)=q^{2}(t ; 1 / \lambda, 0, T) ; \quad q_{3}(t)=q^{3}(t ; 1 / \lambda, 0, T) ; \\
q_{23}(t)= & q^{2}\left(t ; 1 / \lambda, T_{23}, T\right) I_{\left[T_{23}, T\right]}+q^{3}\left(t ; \underline{c}^{p-1}, 0, T_{23}\right) I_{\left[0, T_{23}\right]} ; \\
q_{21}(t)= & q^{2}\left(t ; 1 / \lambda, T_{21}, T\right) I_{\left[T_{21}, T\right]}+q^{1}\left(t ; \bar{c}^{p-1}, 0, T_{21}\right) I_{\left[0, T_{21}\right]} ; \\
q_{32}(t)= & q^{3}\left(t ; 1 / \lambda, T_{32}, T\right) I_{\left[T_{32}, T\right]}+q^{2}\left(t ; \underline{c}^{p-1}, 0, T_{32}\right) I_{\left[0, T_{32}\right]} ;
\end{aligned}
$$

4 In the case of $\underline{c}=0$, the results are similar to those in Table 8 except that $q_{123}$ and $q_{23}$ are replaced by $q_{12}$ and $q_{2}$, respectively. Note that in this case, the forth and fifth rows in Table 8 and $q_{123}, q_{23}, q_{32}, q_{321}, q^{3}, T_{123}, T_{23}, T_{32}, T_{321}$ are irrelevant. 


$$
\begin{aligned}
q_{321}(t)= & q^{3}\left(t ; 1 / \lambda, T_{32}, T\right) I_{\left[T_{32}, T\right]}+q^{2}\left(t ; \underline{c}^{p-1}, T_{321}, T_{32}\right) I_{\left[T_{321}, T_{32}\right]} \\
& +q^{1}\left(t ; \bar{c}^{p-1}, 0, T_{321}\right) I_{\left[0, T_{321}\right]},
\end{aligned}
$$

where $I_{[T, \bar{T}]}$ is an indicator function of the set $[\underline{T}, \bar{T}]$, and the functions $q^{1}(t ; A, \underline{T}, \bar{T}), q^{2}$ $(t ; A, \underline{T}, \bar{T}), q^{3}(t ; A, \underline{T}, \bar{T})$ in the interval $[\underline{T}, \bar{T}]$ are given as

$$
\begin{aligned}
& q^{1}(t ; A, \underline{T}, \bar{T}) \\
& \quad=\ln \lambda+ \begin{cases}\ln \left[\left(A-\frac{\bar{c}^{p}}{\rho+p \bar{c}-p K}\right) e^{(\rho+p \bar{c}-p K)(t-\bar{T})}+\frac{\bar{c}^{p}}{\rho+p \bar{c}-p K}\right], & \rho-p K \neq-p \bar{c} \\
\ln \left[A+\bar{c}^{p}(\bar{T}-t)\right], & \rho-p K=-p \bar{c}\end{cases}
\end{aligned}
$$$$
q^{2}(t ; A, \underline{T}, \bar{T})
$$$$
=\ln \lambda+ \begin{cases}(1-p) \ln \left[\left(A^{1 /(1-p)}-\frac{1-p}{\rho-p K}\right) e^{\frac{\rho-p K}{1-p}(t-\bar{T})}+\frac{1-p}{\rho-p K}\right], & \rho-p K \neq 0 ; \\ (1-p) \ln \left[A^{1 /(1-p)}+\bar{T}-t\right], & \rho-p K=0\end{cases}
$$

$q^{3}(t ; A, \underline{T}, \bar{T})$

$$
=\ln \lambda+ \begin{cases}\ln \left[\left(A-\frac{\underline{c}^{p}}{\rho+p \underline{\underline{c}}-p K}\right) e^{(\rho+p \underline{c}-p K)(t-\bar{T})}+\frac{\underline{c}^{p}}{\rho+p \underline{c}-p K}\right], & \rho-p K \neq-p \underline{c} \\ \ln \left[A+\underline{c}^{p}(\bar{T}-t)\right], & \rho-p K=-p \underline{c}\end{cases}
$$

and $T_{12}, T_{123}, T_{23}, T_{21}, T_{32}, T_{321}$ are given as

$$
\begin{aligned}
& T_{12}= \begin{cases}T+\frac{1}{\rho+p \bar{c}-p K}\left[\ln \left|\bar{c}^{p-1}-\frac{\bar{c}^{p}}{\rho+p \bar{c}-p K}\right|-\ln \left|\frac{1}{\lambda}-\frac{\bar{c}^{p}}{\rho+p \bar{c}-p K}\right|\right], & \rho-p K \neq-p \bar{c} ; \\
T-1 / \bar{c}+1 /\left(\lambda \bar{c}^{p}\right), & \rho-p K=-p \bar{c}\end{cases} \\
& T_{123}= \begin{cases}T_{12}+\frac{1-p}{\rho-p K}\left[\ln \left|\frac{1}{\underline{c}}-\frac{1-p}{\rho-p K}\right|-\ln \left|\frac{1}{\bar{c}}-\frac{1-p}{\rho-p K}\right|\right], \quad \rho-p K \neq 0 \\
T_{12}+1 / \bar{c}-1 / \underline{c}, & \rho-p K=0\end{cases} \\
& T_{23}= \begin{cases}T+\frac{1-p}{\rho-p K}\left[\ln \left|\frac{1}{\underline{c}}-\frac{1-p}{\rho-p K}\right|-\ln \left|\lambda^{1 /(p-1)}-\frac{1-p}{\rho-p K}\right|\right], \quad \rho-p K \neq 0 \\
T+\lambda^{1 /(p-1)}-1 / \underline{c},\end{cases} \\
& T_{21}=T+\frac{1-p}{\rho-p K}\left[\ln \left(\frac{1}{\bar{c}}-\frac{1-p}{\rho-p K}\right)-\ln \left(\lambda^{1 /(p-1)}-\frac{1-p}{\rho-p K}\right)\right]
\end{aligned}
$$


It is routine to check that for any $A>0$ and $0 \leq \underline{T} \leq \bar{T}$, the functions $q^{1}(t ; A, \underline{T}, \bar{T})$, $q^{2}(t ; A, \underline{T}, \bar{T})$ and $q^{3}(t ; A, \underline{T}, \bar{T})$ solve the following ODEs, respectively,

$$
\begin{aligned}
& q_{P}(t)=\ln \lambda+\ln A+\int_{t}^{\bar{T}}\left[-\rho+\lambda \bar{c}^{p} e^{-q_{P}(s)}-p \bar{c}+p K\right] d s, \forall t \in[\underline{T}, \bar{T}] ; \\
& q_{P}(t)=\ln \lambda+\ln A+\int_{t}^{\bar{T}}\left[-\rho+(1-p) \lambda^{\frac{1}{(1-p)}} \exp \left\{\frac{q_{P}(s)}{p-1}\right\}+p K\right] d s, \forall t \in[\underline{T}, \bar{T}] ;
\end{aligned}
$$

$$
q_{P}(t)=\ln \lambda+\ln A+\int_{t}^{\bar{T}}\left[-\rho+\lambda \underline{c}^{p} e^{-q_{P}(s)}-p \underline{c}+p K\right] d s, \forall t \in[\underline{T}, \bar{T}] .
$$

When $\underline{c}>0, q^{1}(0 ; A, 0, \bar{T}), q^{2}(0 ; A, 0, \bar{T})$ and $q^{3}(0 ; A, 0, \bar{T})$ have the following asymptotic properties,

$$
\begin{aligned}
& \lim _{\bar{T} \rightarrow \infty} q^{1}(0 ; A, 0, \bar{T})= \begin{cases}\ln \lambda+\ln \left(\frac{\bar{c}^{p}}{\rho+p \bar{c}-p K}\right), & \rho-p K>-p \bar{c} ; \\
+\infty, & \rho-p K \leq-p \bar{c}\end{cases} \\
& \lim _{\bar{T} \rightarrow \infty} q^{1}(0 ; A, 0, \bar{T}) \leq(p-1) \ln \bar{c}+\ln \lambda \Leftrightarrow \rho-p K \geq(1-p) \bar{c} ; \\
& \lim _{\bar{T} \rightarrow \infty} q^{2}(0 ; A, 0, \bar{T})= \begin{cases}\ln \lambda+(1-p) \ln \left(\frac{1-p}{\rho-p K}\right), & \rho-p K>0 ; \\
+\infty, & \rho-p K \leq 0 ;\end{cases} \\
& \lim _{\bar{T} \rightarrow \infty} q^{2}(0 ; A, 0, \bar{T}) \geq(p-1) \ln \bar{c}+\ln \lambda \Leftrightarrow \rho-p K \leq(1-p) \bar{c} ; \\
& \lim _{\bar{T} \rightarrow \infty} q^{2}(0 ; A, 0, \bar{T}) \leq(p-1) \ln \underline{c}+\ln \lambda \Leftrightarrow \rho-p K \geq(1-p) \underline{c} ; \\
& \lim _{\bar{T} \rightarrow \infty} q^{3}(0 ; A, 0, \bar{T})= \begin{cases}\ln \lambda+\ln \left(\frac{\underline{c}^{p}}{\rho+p \underline{c}-p K}\right), & \rho-p K>-p \underline{c} ; \\
+\infty, & \rho-p K \leq-p \underline{c} ;\end{cases} \\
& \lim _{\bar{T} \rightarrow \infty} q^{3}(0 ; A, 0, \bar{T}) \geq(p-1) \ln \underline{c}+\ln \lambda \Leftrightarrow \rho-p K \leq(1-p) \underline{c} .
\end{aligned}
$$

Proof of Theorem 4.5 Case (1) $0 \leq \underline{c}<\bar{c}<\lambda^{1 /(1-p)}$.

In this case, $(p-1) \ln \bar{c}+\ln \lambda>0$. Since $q_{P}(T)=0$, then, when $t$ is close to $T$, $q_{P}(t)<(p-1) \ln \bar{c}+\ln \lambda$ and $c^{*}(t)=\tilde{x}_{c, P}^{*}\left(q_{P}(t)\right)=\bar{c}$. Thus $q_{P}(t)$ satisfies ODE (46) with $A=1 / \lambda$ and $\bar{T}=T$ in the interval $[\underline{T}, T]$, until $\underline{T}=0$ or $q_{P}(\underline{T})=(p-1) \ln \bar{c}+\ln \lambda$.

(1.1) If $\rho-p K<(1-p) \underline{c} \neq 0$, solving ODE (46), we obtain $q_{P}(t)=q^{1}(t ; 1 / \lambda, \underline{T}, T)$ taking the form of (37) in the interval $[\underline{T}, T]$. According to (49), $q^{1}(0 ; 1 / \lambda, 0, T)>(p-$ 1) $\ln \bar{c}+\ln \lambda$ provided $T$ is large enough. Thus, there exists a positive constant $T_{12}$ such that $q^{1}\left(T_{12} ; 1 / \lambda, T_{12}, T\right)=(p-1) \ln \bar{c}+\ln \lambda$, and $T_{12}$ is given in (40). Hence, we derive $q_{P}(t)=q^{1}\left(t ; 1 / \lambda, T_{12}, T\right)$, and $c^{*}(t)=\bar{c}$ in the interval $\left[T_{12}, T\right]$.

Since

$$
q_{P}^{\prime}\left(T_{12}\right)=\rho-p f_{P}\left(q_{P}\left(T_{12}\right), c^{*}\left(T_{12}\right)\right)-p g\left(x_{\pi}^{*} ; x_{\mu}^{*}, x_{\sigma}^{*}\right)=\rho-(1-p) \bar{c}-p K<0,
$$

then, when $t<T_{12}$ and $t$ is close to $T_{12},(p-1) \ln \bar{c}+\ln \lambda<q_{P}(t)<(p-1) \ln \underline{c}+\ln \lambda$, and $c^{*}(t)=\widehat{c}(t)$ taking the form of (27). Thus $q_{P}(t)$ satisfies ODE (47) with $A=\bar{c}^{p-1}$ and $\bar{T}=T_{12}$ in the interval $\left[\underline{T}, T_{12}\right]$, until $\underline{T}=0$ or $q_{P}(\underline{T})=(p-1) \ln \underline{c}+\ln \lambda$ (where we have used the fact that the sign of $q_{P}^{\prime}(t)$ does not change (cf. Proposition 4.6), and $q_{P}(t)>(p-1) \ln \bar{c}+\ln \lambda$ for any $\left.t<T_{12}\right)$. 
Solving ODE (47), we obtain $q_{P}(t)=q^{2}\left(t ; \bar{c}^{p-1}, \underline{T}, T_{12}\right)$ taking the form of (38) in the interval [ $\underline{T}, T_{12}$ ]. According to $(51), q^{2}\left(0 ; \bar{c}^{p-1}, 0, T_{12}\right)>(p-1) \ln c+\ln \lambda$ provided $T$ is large enough. Thus, there exists a positive constant $T_{123}$ such that $q^{2}\left(T_{123} ; \bar{c}^{p-1}, T_{123}, T_{12}\right)=$ $(p-1) \ln \underline{c}+\ln \lambda$, and $T_{123}$ is given in (41). Hence, we derive $q_{P}(t)=q^{2}\left(t ; \bar{c}^{p-1}, T_{123}, T_{12}\right)$, and $c^{*}(t)=\widehat{c}(t)$ in the interval $\left[T_{123}, T_{12}\right]$.

Recalling the fact that the sign of $q_{P}^{\prime}(t)$ does not change (cf. Proposition 4.6), we deduce that $q_{P}(t) \geq(p-1) \ln \underline{c}+\ln \lambda, c^{*}(t)=\underline{c}$, and $q_{P}(t)$ satisfies ODE (48) with $A=\underline{c}^{p-1}$ and $\bar{T}=T_{123}$ in the interval $\left[0, T_{123}\right]$. Solving ODE (48), we have $q_{P}(t)=q^{3}\left(t ; \underline{c}^{p-1}, 0, T_{123}\right)$ as in (39).

(1.2) If $\rho-p K<(1-p) \underline{c}=0$ or $(1-p) \underline{c} \leq \rho-p K<(1-p) \bar{c}$, repeating the same argument as in Case (1.1), we have $q_{P}(t)=q^{1}\left(t ; 1 / \lambda, T_{12}, T\right)$ as in (37), and $c^{*}=\bar{c}$ in the interval $\left[T_{12}, T\right]$, and $q_{P}(t)=q^{2}\left(t ; \bar{c}^{p-1}, \underline{T}, T_{12}\right)$ as in (38) until $\underline{T}=0$ or $q_{P}(\underline{T})=$ $(p-1) \ln \underline{c}+\ln \lambda$.

In the case of $\rho-p K \leq(1-p) \underline{c}=0,(p-1) \ln \underline{c}+\ln \lambda=+\infty>q^{2}\left(t ; \bar{c}^{p-1}, 0, T_{12}\right)$, and $\underline{T}=0$. In the other case, since $\rho-p K>0$ and $\rho-p K-(1-p) \bar{c}<0$, then (38) implies that

$$
\begin{aligned}
q^{2}\left(t ; \bar{c}^{p-1}, 0, T_{12}\right) & <\ln \lambda+(1-p) \ln \frac{1-p}{\rho-p K} \\
& \leq \ln \lambda+(1-p) \ln \frac{1}{\underline{c}}=(p-1) \ln \underline{c}+\ln \lambda, \quad \forall t \in\left[0, T_{12}\right] .
\end{aligned}
$$

Thus, we deduce that $\underline{T}=0$. Therefore, $q_{P}(t)=q^{2}\left(t ; \bar{c}^{p-1}, 0, T_{12}\right)$ and $c^{*}(t)=\widehat{c}(t)$ in the interval $\left[0, T_{12}\right]$.

(1.3) If $(1-p) \bar{c} \leq \rho-p K<\lambda \bar{c}^{p}-p \bar{c}$, solving ODE (46), we have $q_{P}(t)=q^{1}(t ; 1 / \lambda, \underline{T}, T)$ as in (37) until $\underline{T}=0$ or $q_{P}(\underline{T})=(p-1) \ln \bar{c}+\ln \lambda$. Since

$$
\begin{aligned}
q^{1}(t ; 1 / \lambda, 0, T) & <\ln \lambda+\ln \frac{\bar{c}^{p}}{\rho+p \bar{c}-p K} \\
& \leq \ln \lambda+\ln \bar{c}^{p-1}=(p-1) \ln \bar{c}+\ln \lambda, \quad \forall t \in[0, T],
\end{aligned}
$$

then $q_{P}(t)=q^{1}(t ; 1 / \lambda, 0, T)$ and $c^{*}(t)=\bar{c}$ in the interval $[0, T]$.

(1.4) If $\rho-p K \geq \lambda \bar{c}^{p}-p \bar{c}$, solving ODE (46), we derive that $q_{P}(t)=q^{1}(t ; 1 / \lambda, \underline{T}, T)$ until $\underline{T}=0$ or $q_{P}(\underline{T})=(p-1) \ln \bar{c}+\ln \lambda$.

Since $\rho+p \bar{c}-p K \geq 0$ and $\rho+p \bar{c}-p K-\lambda \bar{c}^{p} \geq 0$, then $q^{1}(t ; 1 / \lambda, 0, T)$ is nondecreasing with respect to $t$, thus for $t \in[0, T], q^{1}(t ; 1 / \lambda, 0, T) \leq q^{1}(T ; 1 / \lambda, 0, T)=0<(p-$ 1) $\ln \bar{c}+\ln \lambda$. Hence, $q_{P}(t)=q^{1}(t ; 1 / \lambda, 0, T)$ and $c^{*}(t)=\bar{c}$ in the interval $[0, T]$.

Case (2) $0 \leq \underline{c}<\bar{c}=\lambda^{1 /(1-p)}$. In this case, note that $(p-1) \ln \bar{c}+\ln \lambda=0$.

(2.1) If $\rho-p K<(1-p) \underline{c} \neq 0$, since

$$
\begin{aligned}
q_{P}^{\prime}(T-0) & =\rho-p f_{P}\left(q_{P}(T), c^{*}(T)\right)-p g\left(x_{\pi}^{*} ; x_{\mu}^{*}, x_{\sigma}^{*}\right) \\
& =\rho-(1-p) \bar{c}-p K \leq \rho-p K-(1-p) \underline{c}<0,
\end{aligned}
$$

then, when $t$ is close to $T, q_{P}(t)>0=(p-1) \ln \bar{c}+\ln \lambda, q_{P}(t)<(p-1) \ln \underline{c}+\ln \lambda$, and $c^{*}(t)=\widehat{c}(t)$. Thus $q_{P}(t)$ satisfies ODE (47) with $A=1 / \lambda$ and $\bar{T}=T$ in the interval $[\underline{T}, T]$, until $\underline{T}=0$ or $q_{P}(\underline{T})=(p-1) \ln \underline{c}+\ln \lambda$.

Solving ODE (47), we obtain $q_{P}(t)=q^{2}(t ; 1 / \lambda, \underline{T}, T)$ as in (38) in the interval [ $\underline{T}, T$ ]. According to $(51), q^{2}(0 ; 1 / \lambda, 0, T)>(p-1) \ln \underline{c}+\ln \lambda$ provided that $T$ is large enough. Thus, there exists a positive constant $T_{23}$ such that $\bar{q}^{2}\left(T_{23} ; 1 / \lambda, T_{23}, T\right)=(p-1) \ln \underline{c}+\ln \lambda$, and $T_{23}$ is given in (42). Hence, we derive that $q_{P}(t)=q^{2}\left(t ; 1 / \lambda, T_{23}, T\right)$, and $c^{*}(t)=\widehat{c}(t)$ in the interval [ $T_{23}, T$ ]. 
Recalling the fact that the sign of $q_{P}^{\prime}(t)$ does not change, we deduce that in the interval $\left[0, T_{23}\right], q_{P}(t) \geq q_{P}\left(T_{23}\right)=(p-1) \ln \underline{c}+\ln \lambda, c^{*}(t)=\underline{c}$, and $q_{P}(t)$ satisfies ODE (48) with $A=\underline{c}^{p-1}$ and $\bar{T}=T_{23}$. Solving ODE (48), we have $q_{P}(t)=q^{3}\left(t ; \underline{c}^{p-1}, 0, T_{23}\right)$ as in (39) in the interval $\left[0, T_{23}\right]$.

(2.2) If $\rho-p K<(1-p) \underline{c}=0$ or $(1-p) \underline{c} \leq \rho-p K<(1-p) \bar{c}$, since

$$
q_{P}^{\prime}(T-0)=\rho-p f_{P}\left(q_{P}(T), c^{*}(T)\right)-p g\left(x_{\pi}^{*} ; x_{\mu}^{*}, x_{\sigma}^{*}\right)=\rho-(1-p) \bar{c}-p K<0
$$

still holds, then repeating the similar argument as in Case (2.1), we deduce that $c^{*}(t)=\widehat{c}(t)$ and $q_{P}(t)=q^{2}(t ; 1 / \lambda, \underline{T}, T)$ in the interval $[\underline{T}, T]$, until $\underline{T}=0$ or $q_{P}(\underline{T})=(p-1) \ln \underline{c}+$ $\ln \lambda$.

In the case of $\rho-p K \leq(1-p) \underline{c}=0,(p-1) \ln \underline{c}+\ln \lambda=+\infty>q^{2}(t ; 1 / \lambda, 0, T)$, and $\underline{T}=0$. In the other case, since $\rho-p K>0$ and $(\rho-p K)-(1-p) \lambda^{1 /(1-p)}=$ $(\rho-p K)-(1-p) \bar{c}<0$, then for any $t \in[0, T]$, we still have

$$
q^{2}(t ; 1 / \lambda, 0, T)<\ln \lambda+(1-p) \ln \frac{1-p}{\rho-p K} \leq \ln \lambda+(1-p) \ln \frac{1}{\underline{c}}=(p-1) \ln \underline{c}+\ln \lambda .
$$

Therefore, $q_{P}(t)=q^{2}(t ; 1 / \lambda, 0, T)$ and $c^{*}(t)=\widehat{c}(t)$ in the interval $[0, T]$.

(2.3) If $\rho-p K \geq(1-p) \bar{c}$. We first discuss the case when $\rho-p K>(1-p) \bar{c}$. Combining the following calculation

$$
q_{P}^{\prime}(T-0)=\rho-p f_{P}\left(q_{P}(T), c^{*}(T)\right)-p g\left(x_{\pi}^{*} ; x_{\mu}^{*}, x_{\sigma}^{*}\right)=\rho-(1-p) \bar{c}-p K>0,
$$

and the fact that the sign of $q_{P}^{\prime}(t)$ does not change, we drive that $q_{P}(t)<q_{P}(T)=0=$ $(p-1) \ln \bar{c}+\ln \lambda, c^{*}(t)=\bar{c}$, and $q_{P}(t)$ satisfies ODE (46) with $A=1 / \lambda$ and $\bar{T}=T$ in the interval $[0, T]$. Solving ODE (46), we have $q_{P}(t)=q^{1}(t ; 1 / \lambda, 0, T)$ and $c^{*}(t)=\bar{c}$ in the interval $[0, T]$.

On the other hand, if $\rho-p K=(1-p) \bar{c}$, then $\rho-p K=\lambda \bar{c}^{p}-p \bar{c}$, and for $t \in[0, T]$, we have $q_{P}(t)=0$, thus still have $q_{P}(t)=q^{1}(t ; 1 / \lambda, 0, T)$.

Case (3) $0 \leq \underline{c}<\lambda^{1 /(1-p)}<\bar{c}$.

In this case, note that $(p-1) \ln \bar{c}+\ln \lambda<0<(p-1) \ln \underline{c}+\ln \lambda$. Since $q_{P}(T)=0$, then, when $t$ is close to $T,(p-1) \ln \bar{c}+\ln \lambda<q_{P}(t)<(p-1) \ln \underline{c}+\ln \lambda, c^{*}(t)=\widehat{c}(t)$ and $q_{P}(t)$ satisfies ODE (47) with $A=1 / \lambda$ and $\bar{T}=T$ in the interval $[\underline{T}, T]$, until $\underline{T}=0$ or $q_{P}(\underline{T})=(p-1) \ln \bar{c}+\ln \lambda$ or $q_{P}(\underline{T})=(p-1) \ln \underline{c}+\ln \lambda$.

(3.1) If $\rho-p K<(1-p) \underline{c} \neq 0$, solving ODE (47), we have $q_{P}(t)=q^{2}(t ; 1 / \lambda, \underline{T}, T)$ and $c^{*}(t)=\widehat{c}(t)$ in the interval $[\underline{T}, T]$.

Since

$$
\begin{aligned}
q_{P}^{\prime}(T-0) & =\rho-p f_{P}\left(q_{P}(T), c^{*}(T)\right)-p g\left(x_{\pi}^{*} ; x_{\mu}^{*}, x_{\sigma}^{*}\right) \\
& =\rho-(1-p) \lambda^{1 /(1-p)}-p K<\rho-(1-p) \underline{c}-p K<0,
\end{aligned}
$$

then we deduce $q_{P}(t)$ is nonincreasing with respect to $t$ from the fact that the sign of $q_{P}^{\prime}(t)$ does not change. Hence, we have $q_{P}(t)>(p-1) \ln \bar{c}+\ln \lambda$ for any $t \in[0, T]$. Moreover, (51) implies that $q^{2}(0 ; 1 / \lambda, 0, T)>(p-1) \ln \underline{c}+\ln \lambda$ provided that $T$ is large enough. Thus, there exists a positive constant $T_{23}$ such that $q^{2}\left(T_{23} ; 1 / \lambda, T_{23}, T\right)=(p-1) \ln \underline{c}+\ln \lambda$, and $T_{23}$ is given in (42). Hence, we derive that $q_{P}(t)=q^{2}\left(t ; 1 / \lambda, T_{23}, T\right)$, and $c^{*}(t)=\widehat{c}(t)$ in the interval $\left[T_{23}, T\right]$.

Since

$$
q_{P}^{\prime}\left(T_{23}\right)=\rho-p f_{P}\left(q_{P}\left(T_{23}\right), c^{*}\left(T_{23}\right)\right)-p g\left(x_{\pi}^{*} ; x_{\mu}^{*}, x_{\sigma}^{*}\right)=\rho-(1-p) \underline{c}-p K<0,
$$


then for any $t \in\left[0, T_{23}\right)$, we have $q_{P}(t)>(p-1) \ln \underline{c}+\ln \lambda$, and $q_{P}(t)$ satisfies ODE (48) with $A=\underline{c}^{p-1}$ and $\bar{T}=T_{23}$ in the interval [0, $T_{23}$ ]. Solving ODE (48), we obtain $q_{P}(t)=q^{3}\left(t ; \underline{c}^{\bar{p}-1}, 0, T_{23}\right)$ and $c^{*}(t)=\underline{c}$ in the interval $\left[0, T_{23}\right]$.

(3.2) If $\rho-p \bar{K}<(1-p) \underline{c}=0$ or $(1-p) \underline{c} \leq \rho-p K<(1-p) \lambda^{1 /(1-p)}$, repeating the similar argument as in case (3.1), we deduce that $q_{P}(t)=q^{2}(t ; 1 / \lambda, \underline{T}, T), c^{*}(t)=\widehat{c}(t)$ in the interval $[\underline{T}, T]$, until $\underline{T}=0$ or $q_{P}(\underline{T})=(p-1) \ln \underline{c}+\ln \lambda$.

For the case of $\rho-p \bar{K} \leq(1-p) \underline{c}=0,(p-1) \ln \underline{c}+\ln \lambda=+\infty>q^{2}(t ; 1 / \lambda, 0, T)$, and $T=0$. For the other case, since $\rho-p K>0$ and $\rho-p K-(1-p) \lambda^{1 /(1-p)}<0$, then (53) still holds. Therefore, $q_{P}(t)=q^{2}(t ; 1 / \lambda, 0, T)$ and $c^{*}(t)=\widehat{c}(t)$ in the interval $[0, T]$.

(3.3) If $(1-p) \lambda^{1-p} \leq \rho-p K \leq(1-p) \bar{c}$, solving ODE (47), we have $q_{P}(t)=$ $q^{2}(t ; 1 / \lambda, \underline{T}, T)$ and $c^{*}(t)=\widehat{c}(t)$ in the interval $[\underline{T}, T]$. Since $\rho-p K>0$ and $\rho-p K-(1-p) \lambda^{1-p} \geq 0$, then $q^{2}(t ; 1 / \lambda, 0, T)$ is nondecreasing and for $t \in[0, T]$, we have

$$
\begin{aligned}
(p-1) \ln \underline{c}+\ln \lambda & >0=q^{2}(T ; 1 / \lambda, 0, T) \geq q^{2}(t ; 1 / \lambda, 0, T) \\
& \geq(1-p) \ln \left(\frac{1-p}{\rho-p K}\right)+\ln \lambda \\
& \geq(1-p) \ln \frac{1}{\bar{c}}+\ln \lambda=(p-1) \ln \bar{c}+\ln \lambda .
\end{aligned}
$$

Therefore, $q_{P}(t)=q^{2}(t ; 1 / \lambda, 0, T)$ and $c^{*}(t)=\widehat{c}(t)$ in the interval $[0, T]$.

(3.4) If $\rho-p K>(1-p) \bar{c}$, solving ODE (47), we have $q_{P}(t)=q^{2}(t ; 1 / \lambda, \underline{T}, T)$ and $c^{*}(t)=\widehat{c}(t)$ in the interval $[\underline{T}, T]$.

Since

$$
\begin{aligned}
q_{P}^{\prime}(T-0) & =\rho-p f_{P}\left(q_{P}(T), c^{*}(T)\right)-p g\left(x_{\pi}^{*} ; x_{\mu}^{*}, x_{\sigma}^{*}\right) \\
& =\rho-(1-p) \lambda^{1 /(1-p)}-p K>\rho-(1-p) \bar{c}-p K>0,
\end{aligned}
$$

then we deduce $q_{P}(t)$ is nondecreasing with respect to $t$ from the fact that the sign of $q_{P}^{\prime}(t)$ does not change. Hence, we have $q_{P}(t)<(p-1) \ln \underline{c}+\ln \lambda$ for any $t \in[0, T]$. Moreover, (50) implies that $q^{2}(0 ; 1 / \lambda, 0, T)<(p-1) \ln \bar{c}+\ln \lambda$ provided that $T$ is large enough. Thus, there exists a positive constant $T_{21}$ such that $q^{2}\left(T_{21} ; 1 / \lambda, T_{21}, T\right)=(p-1) \ln \bar{c}+\ln \lambda$, and $T_{21}$ is given in (43). Hence, we derive that $q_{P}(t)=q^{2}\left(t ; 1 / \lambda, T_{21}, T\right)$, and $c^{*}(t)=\widehat{c}(t)$ in the interval $\left[T_{21}, T\right]$.

Since

$$
q_{P}^{\prime}\left(T_{21}\right)=\rho-p f_{P}\left(q_{P}\left(T_{21}\right), c^{*}\left(T_{21}\right)\right)-p g\left(x_{\pi}^{*} ; x_{\mu}^{*}, x_{\sigma}^{*}\right)=\rho-(1-p) \bar{c}-p K>0,
$$

then for any $t \in\left[0, T_{12}\right)$, we have $q_{P}(t)<(p-1) \ln \bar{c}+\ln \lambda$, and $q_{P}(t)$ satisfies ODE (46) with $A=\bar{c}^{p-1}$ and $\bar{T}=T_{12}$ in the interval [0, $T_{12}$ ]. Solving ODE (46), we obtain $q_{P}(t)=q^{1}\left(t ; \bar{c}^{p-1}, 0, T_{12}\right)$ and $c^{*}(t)=\bar{c}$ in the interval $\left[0, T_{12}\right]$.

Case (4) $\lambda^{1 /(1-p)}=\underline{c}<\bar{c}$. In this case, note that $(p-1) \ln \bar{c}+\ln \lambda<0=(p-1) \ln \underline{c}+\ln \lambda$. (4.1) If $\rho-p K \leq(1-p) \underline{c}$, we first consider the case where $\rho-p K<(1-p) \underline{c}$. Combining the following calculation

$$
q_{P}^{\prime}(T-0)=\rho-p f_{P}\left(q_{P}(T), c^{*}(T)\right)-p g\left(x_{\pi}^{*} ; x_{\mu}^{*}, x_{\sigma}^{*}\right)=\rho-(1-p) \underline{c}-p K<0,
$$

and the fact that the sign of $q_{P}^{\prime}(t)$ does not change, we deduce that $q_{P}(t)>0=(p-$ 1) $\ln \underline{c}+\ln \lambda, c^{*}(t)=\underline{c}$, and $q_{P}(t)$ satisfies ODE (48) with $A=1 / \lambda$ and $\bar{T}=T$ in the 
interval $[0, T]$. Solving ODE (48), we have $q_{P}(t)=q^{3}(t ; 1 / \lambda, 0, T)$ and $c^{*}(t)=\underline{c}$ in the interval $[0, T]$.

When $\rho-p K=(1-p) \underline{c}$, it is easy to see that for $t \in[0, T]$, we have $q_{P}(t)=0$, and we still have $q_{P}(t)$ equal to $q^{3}(t ; 1,0, T)$ and $c^{*}(t)=\underline{c}$ in the interval $[0, T]$.

(4.2) If $(1-p) \underline{c}<\rho-p K \leq(1-p) \bar{c}$, since $q(T)=0=(p-1) \ln \underline{c}+\ln \lambda$, and

$$
q_{P}^{\prime}(T-0)=\rho-p f_{P}\left(q_{P}(T), c^{*}(T)\right)-p g\left(x_{\pi}^{*} ; x_{\mu}^{*}, x_{\sigma}^{*}\right)=\rho-(1-p) \underline{c}-p K>0,
$$

then, when $t$ is close to $T$, we have $(p-1) \ln \bar{c}+\ln \lambda<q_{P}(t)<(p-1) \ln \underline{c}+\ln \lambda$. Thus, $q_{P}(t)$ satisfies $\operatorname{ODE}(47)$ with $A=1 / \lambda$ and $\bar{T}=T$ in the interval $[\underline{T}, T]$, until $\underline{T}=0$ or $q_{P}(\underline{T})=(p-1) \ln \bar{c}+\ln \lambda$ or $q_{P}(\underline{T})=(p-1) \ln \underline{c}+\ln \lambda$. Recalling the fact that the sign of $q_{P}^{\prime}(t)$ does not change, we deduce that $q_{P}(t)$ is nondecreasing with respect to $t$. Thus, it is impossible that $q_{P}(\underline{T})=(p-1) \ln \underline{c}+\ln \lambda$ for some $\underline{T} \in[0, T)$.

Solving ODE (47), we have $q_{P}(t)=q^{2}(t ; 1 / \lambda, \underline{T}, T)$ and $c^{*}(t)=\widehat{c}(t)$ in the interval $[\underline{T}, T]$. Since $(\rho-p K)-(1-p) \lambda^{1 /(1-p)}=(\rho-p K)-(1-p) \underline{c}>0$, then $q^{2}(t ; 1 / \lambda, 0, T)$ is increasing with respect to $t$, thus for $t \in[0, T)$, we have

$$
\begin{aligned}
(p-1) \ln \underline{c}+\ln \lambda & =0=q^{2}(T ; 1 / \lambda, 0, T)>q^{2}(t ; 1 / \lambda, 0, T)>(1-p) \ln \frac{1-p}{\rho-p K}+\ln \lambda \\
& \geq(1-p) \ln \frac{1}{\bar{c}}+\ln \lambda=(p-1) \ln \bar{c}+\ln \lambda .
\end{aligned}
$$

Therefore, $q_{P}(t)=q^{2}(t ; 1 / \lambda, 0, T)$ and $c^{*}(t)=\widehat{c}(t)$ in the interval $[0, T]$.

(4.3) If $\rho-p K>(1-p) \bar{c}$, repeating the similar argument as in case (4.2), we deduce that $q_{P}(t)=q^{2}(t ; 1 / \lambda, \underline{T}, T)$ and $c^{*}(t)=\widehat{c}(t)$ in the interval $[\underline{T}, T]$, until $\underline{T}=0$ or $q_{P}(\underline{T})=(p-1) \ln \bar{c}+\ln \lambda$.

According to (50), $q^{2}(0 ; 1 / \lambda, 0, T)<(p-1) \ln \bar{c}+\ln \lambda$ provided that $T$ is large enough. Thus, there exists a positive constant $T_{21}$ such that $q^{2}\left(T_{21} ; 1 / \lambda, T_{21}, T\right)=(p-1) \ln \bar{c}+\ln \lambda$, and $T_{21}$ is given in (43). Hence, we derive that $q_{P}(t)=q^{2}\left(t ; 1 / \lambda, T_{21}, T\right)$, and $c^{*}(t)=\widehat{c}(t)$ in the interval $\left[T_{21}, T\right]$.

Combining

$$
q_{P}^{\prime}\left(T_{21}\right)=\rho-p f_{P}\left(q_{P}\left(T_{21}\right), c^{*}\left(T_{21}\right)\right)-p g\left(x_{\pi}^{*} ; x_{\mu}^{*}, x_{\sigma}^{*}\right)=\rho-p K-(1-p) \bar{c}>0,
$$

and the fact that the sign of $q_{P}^{\prime}(t)$ does not change, we deduce that in the interval $\left[0, T_{21}\right)$, $q_{P}(t)<(p-1) \ln \bar{c}+\ln \lambda, c^{*}(t)=\bar{c}$, and $q_{P}(t)$ satisfies ODE (46) with $A=\bar{c}^{p-1}$ and $\bar{T}=T_{21}$. Solving ODE (46), we obtain $q_{P}(t)=q^{1}\left(t ; \bar{c}^{p-1}, 0, T_{21}\right)$ in the interval [0, $\left.T_{21}\right]$. Case (5) $\lambda^{1 /(1-p)}<\underline{c}<\bar{c}$.

Since $q_{P}(T)=0>(p-1) \ln \underline{c}+\ln \lambda$, then $q_{P}(t)$ satisfies ODE (48) with $A=1 / \lambda$ and $\bar{T}=T$ in the interval $[\underline{T}, T]$, until $\underline{T}=0$ or $q_{P}(\underline{T})=(p-1) \ln \underline{c}+\ln \lambda$. Solving ODE (48), we obtain $q_{P}(t)=q^{3}(t ; 1 / \lambda, \underline{T}, T)$ and $c^{*}(t)=\underline{c}$ in the interval $[\underline{T}, T]$.

(5.1) If $\rho-p K \leq \lambda \underline{c}^{p}-p \underline{c}$, then $\rho+p \underline{c}-p K-\lambda \underline{c}^{p} \leq 0$, and $q^{3}(t ; 1 / \lambda, 0, T)$ is nonincreasing with respect to $t$, thus for $t \in[0, T]$, we have $q^{3}(t ; 1 / \lambda, 0, T) \geq q^{3}(T ; 1 / \lambda, 0, T)=$ $0>(p-1) \ln \underline{c}+\ln \lambda$. Therefore, $q_{P}(t)=q^{3}(t ; 1 / \lambda, 0, T)$ and $c^{*}(t)=\underline{c}$ in the interval $[0, T]$.

(5.2) If $\lambda \underline{c}^{p}-p \underline{c}<\rho-p K \leq(1-p) \underline{c}$, then $\rho+p \underline{c}-p K-\lambda \underline{c}^{p}>0$, and

$$
\begin{aligned}
q^{3}(t ; 1 / \lambda, 0, T) & \geq \ln \lambda+\ln \frac{\underline{c}^{p}}{\rho+p \underline{c}-p K} \\
& \geq \ln \frac{\underline{c}^{p}}{\underline{c}}+\ln \lambda=(p-1) \ln \underline{c}+\ln \lambda, \quad \forall t \in[0, T] .
\end{aligned}
$$


Therefore we still have $q_{P}(t)=q^{3}(t ; 1 / \lambda, 0, T)$ and $c^{*}(t)=\underline{c}$ in the interval $[0, T]$.

(5.3) If $(1-p) \underline{c}<\rho-p K \leq(1-p) \bar{c}$, then (52) implies that $q^{3}(0 ; 1 / \lambda, 0, T)<(p-$ 1) $\ln \underline{c}+\ln \lambda$ provided that $T$ is large enough. Thus, there exists a positive constant $T_{32}$ such that $\bar{q}^{3}\left(T_{32} ; 1 / \lambda, T_{32}, T\right)=(p-1) \ln \underline{c}+\ln \lambda$, and $T_{32}$ is given in (44). Hence, we derive $q_{P}(t)=q^{3}\left(t ; 1 / \lambda, T_{32}, T\right)$, and $c^{*}(t)=\underline{c}$ in the interval $\left[T_{32}, T\right]$.

Since

$$
q_{P}^{\prime}\left(T_{32}\right)=\rho-p f_{P}\left(q_{P}\left(T_{32}\right), c^{*}\left(T_{32}\right)\right)-p g\left(x_{\pi}^{*} ; x_{\mu}^{*}, x_{\sigma}^{*}\right)=\rho-(1-p) \underline{c}-p K>0,
$$

then, when $t<T_{32}$ and $t$ is close to $T_{32}$, we have $q_{P}(t)<(p-1) \ln \underline{c}+\ln \lambda$ and $q_{P}(t)>(p-$ 1) $\ln \bar{c}+\ln \lambda$, and $q_{P}(t)$ is nondecreasing with respect to $t$, and $q_{P}(t)$ satisfies ODE (47) with $A=\underline{c}^{p-1}$ and $\bar{T}=T_{32}$ in the interval $\left[\underline{T}, T_{32}\right]$, until $\underline{T}=0$ or $q_{P}(\underline{T})=(p-1) \ln \bar{c}+\ln \lambda$. Solving ODE (47), we obtain $q_{P}(t)=\bar{q}^{2}\left(t ; \underline{c}^{p-1}, \underline{T}, T_{32}\right)$ and $c^{*}(t)=\widehat{c}(t)$ in the interval $\left[\underline{T}, T_{32}\right]$.

Since $\rho-p K-(1-p) \lambda^{1 /(1-p)}>\rho-p K-(1-p) \underline{c}>0$, then in this case $q^{2}\left(t ; \underline{c}^{p-1}, 0, T_{32}\right)$ is increasing with respect to $t$, thus for $t \in\left[0, T_{32}\right)$, we have

$$
\begin{aligned}
(p-1) \ln \underline{c}+\ln \lambda & =q^{2}\left(T_{32} ; \underline{c}^{p-1}, 0, T_{32}\right)>q^{2}\left(t ; \underline{c}^{p-1}, 0, T_{32}\right) \\
& >\ln \lambda+(1-p) \ln \frac{1-p}{\rho-p K} \\
& \geq(1-p) \ln \frac{1}{\bar{c}}+\ln \lambda=(p-1) \ln \bar{c}+\ln \lambda .
\end{aligned}
$$

Therefore, $q_{P}(t)=q^{2}\left(t ; \underline{c}^{p-1}, 0, T_{32}\right)$ and $c^{*}(t)=\widehat{c}(t)$ in the interval [0, $\left.T_{32}\right]$.

(5.4) If $\rho-p K>(1-p) \bar{c}$, repeating the similar argument as in case (5.3), we deduce that $q_{P}(t)=q^{3}\left(T_{32} ; 1 / \lambda, T_{32}, T\right)$, and $c^{*}(t)=\underline{c}$ in the interval $\left[T_{32}, T\right]$, and $q_{P}(t)=$ $q^{2}\left(t ; \underline{c}^{p-1}, \underline{T}, T_{32}\right)$ and $c^{*}(t)=\widehat{c}(t)$ in the interval $\left[\underline{T}, T_{32}\right]$, until $\underline{T}=0$ or $q_{P}(\underline{T})=$ $(p-1) \ln \bar{c}+\ln \lambda$.

According to (50), $q^{2}\left(0 ; \underline{c}^{p-1}, 0, T_{32}\right)<(p-1) \ln \bar{c}+\ln \lambda$ provided that $T$ is large enough. Thus, there exists a positive constant $T_{321}$ such that $q^{2}\left(T_{321} ; \underline{c}^{p-1}, T_{321}, T_{32}\right)=(p-1) \ln \bar{c}+$ $\ln \lambda$, and $T_{321}$ is given in (45). Hence, we derive that $q_{P}(t)=q^{2}\left(t ; \underline{c}^{p-1}, T_{321}, T_{32}\right)$, and $c^{*}(t)=\widehat{c}(t)$ in the interval $\left[T_{321}, T_{32}\right]$.

Combining

$q_{P}^{\prime}\left(T_{321}\right)=\rho-p f_{P}\left(q_{P}\left(T_{321}\right), c^{*}\left(T_{321}\right)\right)-p g\left(x_{\pi}^{*} ; x_{\mu}^{*}, x_{\sigma}^{*}\right)=\rho-(1-p) \bar{c}-p K>0$,

and the fact that the sign of $q_{P}^{\prime}(t)$ does not change sign, we deduce that $q_{P}(t)$ is nondecreasing with respect to $t$, and $q_{P}(t)<(p-1) \ln \bar{c}+\ln \lambda$ for any $t \in\left[0, T_{321}\right)$. Thus, $c^{*}(t)=\bar{c}$ and $q_{P}(t)$ satisfies ODE (46) in the interval $t \in\left[0, T_{321}\right)$. Solving ODE (46), we obtain $q_{P}(t)=q^{1}\left(t ; \bar{c}^{p-1}, 0, T_{321}\right)$ in the interval $t \in\left[0, T_{321}\right]$.

\section{References}

1. Bergman, Y.Z.: Option pricing with differential interest rates. Rev. Financ. Stud. 8(2), 475-500 (1995)

2. Biagini, S., Pinar, M.: The robust merton problem of an ambiguity averse investor. Math. Financ. Econ. 11(1), 1-24 (2017)

3. Bo, L., Capponi, A.: Optimal credit investment with borrowing costs. Math. Oper. Res. 42(2), 546-575 (2016)

4. Bordigoni, G., Matoussi, A., Schweizer, M.: A stochastic control approach to a robust utility maximization problem. In: Stochastic Analysis and Applications, Abel Symposium, vol. 2, pp. 125-151 (2007)

5. Briand, P., Delyon, B., Hu, Y., Pardoux, E., Stoica, L.: $L^{p}$ solutions of backward stochastic differential equations. Stoch. Process. Their Appl. 108(1), 109-129 (2003) 
6. Cadenillas, A., Sethi, S.: The consumption-investment problem with subsistence consumption, bankruptcy, and random market coefficients. J. Optim. Theory Appl. 93, 243-272 (1997)

7. Cheridito, P., Hu, Y.: Optimal consumption and investment in incomplete markets with general constraints. Stoch. Dyn. 11, 283-299 (2011)

8. Cvitanić, J., Karatzas, I.: Convex duality in constrained portfolio optimization. Ann. Appl. Probab. 2(4), 767-818 (1992)

9. Denis, L., Kervarec, M.: Optimal investment under model uncertainty in nondominated models. SIAM J. Control Optim. 51(3), 1803-1822 (2013)

10. Epstein, L.G., Ji, S.: Ambiguous volatility, possibility and utility in continuous time. J. Math. Econ. 50(1), 269-282 (2014)

11. Fleming, W.H., Zariphopoulou, T.: An optimal investment/consumption model with borrowing. Math. Oper. Res. 16(4), 802-822 (1991)

12. Fouque, J.P., Pun, C.S., Wong, H.Y.: Portfolio optimization with ambiguous correlation and stochastic volatilities. SIAM J. Control Optim. 54(5), 2309-2338 (2016)

13. Hernández-Hernández, D., Schied, A.: Robust utility maximization in a stochastic factor model. Stat. Decis. 24, 109-125 (2006)

14. Herrmann, S., Muhle-Karbe, J.: Model uncertainty, recalibration, and the emergence of delta-vega hedging. Finance Stoch. 21(4), 873-930 (2017)

15. Hu, Y., Imkeller, P., Muller, M.: Utility maximization in incomplete markets. Ann. Appl. Probab. 15(3), 1691-1712 (2005)

16. Karatzas, I., Lehoczky, J., Sethi, S., Shreve, S.: Explicit solution of a general consumption/investment problem. Math. Oper. Res. 11(2), 261-294 (1986)

17. Karatzas, I., Lehoczky, J., Shreve, S.: Optimal portfolio and consumption decisions for a small investor on a finite horizon. SIAM J. Control Optim. 25(6), 1557-1586 (1987)

18. Jian, X., Yi, F., Zhang, J.: Investment and consumption problem in finite time with consumption constraint. ESAIM: Control Optim. Calc. Var. 23(4), 1601-1615 (2017)

19. Lehoczky, J., Sethi, S., Shreve, S.: Optimal consumption and investment policies allowing consumption constraints and bankruptcy. Math. Oper. Res. 8(4), 613-636 (1983)

20. Lin, Q., Riedel, R.: Optimal consumption and portfolio choice with ambiguity. Preprint arXiv:1401.1639v1 (2014)

21. Matoussi, A., Possamai, D., Zhou, C.: Robust utility maximization in non-dominated models with 2BSDEs: the uncertain volatility model. Math. Finance 25(2), 258-287 (2015)

22. Merton, R.C.: Continuous-Time Finance. Wiley-Blackwell, Oxford (1992)

23. Neufeld, A., Nutz, M.: Robust utility maximization with Lévy processes. Math. Finance 28(1), 82-105 (2018)

24. Nutz, M.: The opportunity process for optimal consumption and investment with power utility. Math. Financ. Econ. 3, 139-159 (2010)

25. Roberts, A.W., Varberg, D.E.: Covex Functions. Academic Press, New York (1973)

26. Schied, A.: Robust optimal control for a consumption-investment problem. Math. Methods Oper. Res. 67, 1-20 (2008)

27. Sethi, S., Taksar, M., Presman, E.: Explicit solution of a general consumption/portfolio problem with subsistence consumption and bankruptcy. J. Econ. Dyn. Control 16, 747-768 (1992)

28. Talay, D., Zheng, Z.: Worst case model risk management. Finance Stoch. 6(4), 517-537 (2002)

29. Tevzadze, R., Toronjadze, T., Uzunashvili, T.: Robust utility maximization for a diffusion market model with misspecified coefficients. Finance Stoch. 17(3), 535-563 (2013)

30. Vila, J.L., Zariphopoulou, T.: Optimal consumption and portfolio choice with borrowing constraints. J. Econ. Theory 77(2), 402-431 (1997)

31. Xu, Z., Yi, F.: An optimal consumption-investment model with constraint on consumption. Math. Control Relat. Fields 6(3), 517-534 (2016)

32. Yan, H., Liang, G., Yang, Z.: Indifference pricing and hedging in a multiple-priors model with trading constraints. Sci. China Math. 58(4), 689-714 (2015)

33. Zariphopoulou, T.: Consumption-investment models with constraints. SIAM J. Control Optim. 32(1), 59-85 (1994)

Publisher's Note Springer Nature remains neutral with regard to jurisdictional claims in published maps and institutional affiliations. 